\title{
RESEARCH
}

Open Access

\section{Unravelling the importance of the eukaryotic and bacterial communities and their relationship with Legionella spp. ecology in cooling towers: a complex network}

Kiran Paranjape ${ }^{1}$, Émilie Bédard ${ }^{2}$, Deeksha Shetty ${ }^{1}$, Mengqi Hu ${ }^{1}$ Fiona Chan Pak Choon ${ }^{1}$, Michèle Prévost ${ }^{2}$ and Sébastien P. Faucher ${ }^{1 *}$ (D)

\begin{abstract}
Background: Cooling towers are a major source of large community-associated outbreaks of Legionnaires' disease, a severe pneumonia. This disease is contracted when inhaling aerosols that are contaminated with bacteria from the genus Legionella, most importantly Legionella pneumophila. How cooling towers support the growth of this bacterium is still not well understood. As Legionella species are intracellular parasites of protozoa, it is assumed that protozoan community in cooling towers play an important role in Legionella ecology and outbreaks. However, the exact mechanism of how the eukaryotic community contributes to Legionella ecology is still unclear. Therefore, we used 185 rRNA gene amplicon sequencing to characterize the eukaryotic communities of 18 different cooling towers. The data from the eukaryotic community was then analysed with the bacterial community of the same towers in order to understand how each community could affect Legionella spp. ecology in cooling towers.

Results: We identified several microbial groups in the cooling tower ecosystem associated with Legionella spp. that suggest the presence of a microbial loop in these systems. Dissolved organic carbon was shown to be a major factor in shaping the eukaryotic community and may be an important factor for Legionella ecology. Network analysis, based on co-occurrence, revealed that Legionella was correlated with a number of different organisms. Out of these, the bacterial genus Brevundimonas and the ciliate class Oligohymenophorea were shown, through in vitro experiments, to stimulate the growth of L. pneumophila through direct and indirect mechanisms.

\footnotetext{
* Correspondence: sebastien.faucher2@mcgill.ca

'Department of Natural Resource Sciences, Faculty of Agricultural and

Environmental Sciences, McGill University, Sainte-Anne-de-Bellevue, QC,

Canada

Full list of author information is available at the end of the article
}

(c) The Author(s). 2020 Open Access This article is licensed under a Creative Commons Attribution 4.0 International License, which permits use, sharing, adaptation, distribution and reproduction in any medium or format, as long as you give appropriate credit to the original author(s) and the source, provide a link to the Creative Commons licence, and indicate if changes were made. The images or other third party material in this article are included in the article's Creative Commons licence, unless indicated otherwise in a credit line to the material. If material is not included in the article's Creative Commons licence and your intended use is not permitted by statutory regulation or exceeds the permitted use, you will need to obtain permission directly from the copyright holder. To view a copy of this licence, visit http://creativecommons.org/licenses/by/4.0/ The Creative Commons Public Domain Dedication waiver (http://creativecommons.org/publicdomain/zero/1.0/) applies to the data made available in this article, unless otherwise stated in a credit line to the data. 
(Continued from previous page)

Conclusion: Our results suggest that Legionella ecology depends on the host community, including ciliates and on several groups of organisms that contribute to its survival and growth in the cooling tower ecosystem. These findings further support the idea that some cooling tower microbiomes may promote the survival and growth of Legionella better than others.

Keywords: 185 rRNA gene amplicon sequencing, Eukaryotic community, Legionella pneumophila, Brevundimonas sp., Dissolved organic carbon, Network analysis, Whole genome sequencing

\section{Background}

Cooling towers are not typically thought of as ecological niches for microorganisms; yet, they harbour a vast quantity of microorganisms [1,2]. A perfect example of their suitability as an ecological niche is that cooling towers are an important source of large community associated outbreaks of Legionnaires' disease (LD), a severe bacterial pneumonia caused by several bacterial species of the genus Legionella, such as Legionella pneumophila [3-5]. Recent outbreaks due to cooling towers have been making headlines in North America, such as the 2015 New York City outbreaks (138 cases, 18 deaths) and the Disneyland outbreak in 2017 (12 reported cases) $[6,7]$. Furthermore, cooling towers are also an important source of sporadic cases of LD [8, 9]. A study from 1978 to 1986 , in the city of Glasgow, Scotland, revealed that around $28 \%$ of sporadic LD cases were associated with cooling towers [8]. The distance from the cooling tower is also believed to be an important risk factor $[8,9]$. Due to their design, cooling towers produce high quantities of aerosols, which when contaminated with L. pneumophila, can spread the bacterium to the surrounding environment, reportedly as far as $12 \mathrm{~km}$ [10]. Individuals in the dispersion area inhaling the aerosols are at risk of infection, with risk increasing as the distance from the source decreases [9].

The Centre for Disease Control in the USA and the European Centre for Disease Prevention and Control have both reported increasing trends of $\mathrm{LD}$ in recent years [11-14]. For instance, the rate of incidence of LD in the USA increased from 0.42 cases per 100,000, in 2000 , to 1.89 cases per 100,000, in 2015 (4.5-fold increase) $[14,15]$. In the European Union, the incidence of reported cases increased by 50\% from 2013 to 2017, with a reported incidence of 1.2 per 100,000 , in 2013 , to an incidence of 1.8 per 100,000 , in 2017 [11]. The exact minimum infectious dose of L. pneumophila is currently a subject of debate. Quantitative microbial risk assessment models have been used to predict the risk of pneumonia associated with aerosolization of Legionella from different engineered water systems $[16,17]$. In an outbreak associated with whirlpool spas, the model showed that an estimated dose of 35 CFU of Legionella may pose a severe clinical risk $[17,18]$. Even with such a low infectious dose, it is clear that the L. pneumophila population must increase within a cooling tower to a highenough threshold, so that sufficient contaminated aerosols are emitted to reach neighbouring population and cause illness. Consequently, understanding growth factors and ecology within cooling towers is of vital importance.

Legionella spp. are intracellular parasites of various protozoan species, such as amoebae and ciliates, and require these host for growth in water systems [19-22]. These host species are microbial grazers that feed on microbial communities. Legionella species have taken advantage of this trait by allowing their phagocytosis and then creating a suitable environment for replication within the phagosome of the host cell. This is achieved through the use of a type IV secretion system that translocates many effector proteins into the intracellular host environment [23]. More than 18,000 effector proteins are present in the pangenome of Legionella [24]. Different species or strain of Legionella can infect different host species, depending on the type of effector protein present in the genome [24]. In addition, host cells provide a safe means of transportation and protection from harsh chemicals, such as disinfectants found in the cooling tower environment, as some protozoa host can produce cysts [25-27]. The number of known host species is quite expansive, spanning several distant phylogenetic groups. For instance, Acanthamoeba castellanii, Naegleria fowleri, Tetrahymena pyriformis and human macrophages are host species belonging to different eukaryotic groups (respectively Amoebozoa, Heterolobosea, Ciliophora, Chordata) and are routinely used as host models for research [28]. Consequently, host diversity and abundance, and the factors influencing them in the cooling tower environment is an important aspect for Legionella survival, proliferation and transmission to humans.

Protozoan host species inhabit various engineered water systems, including cooling towers [29-31]. In these systems, the protozoa usually feed by grazing on a diversity of prey from the bacterial, algal, fungal and other protozoan communities [32, 33]. Several factors can influence the health and proliferation of the protozoan community. Firstly, the species and abundance of the prey community is an important factor for growth of 
the protozoan host population. Grazing is usually a selective process that depends on the prey species, prey morphology, prey size and physiological state of the prey and of the predator [34-36]. As a result, factors affecting the prey community will have consequences on the Legionella host community, and therefore, indirectly on the Legionella community. Accordingly, competition between prey species and non-prey species, competition by predation for the same prey by the non-Legionella host community and chemical and physical parameters of the environments are all elements that can have negative effects on the prey community [37-39]. For instance, Bdellovibrio spp. are bacterial parasites of different bacterial species that could potentially reduce the prey community [40]. Secondly, factors directly affecting the growth and survival of the host community may also affect the Legionella community. Predation by other eukaryotes, parasitism by the bacterial and viral communities and amensal relations with different organisms are direct biological interactions that could negatively affect the host community. For instance, Pseudomonas aeruginosa is known to kill the host amoeba Acanthamoeba castellanii using a type III secretion system and several toxins [41]. On the other hand, symbiotic and mutualistic relations can positively affect the host community. For example, certain species of Parachlamydia and Candidatus Procabacter are known endosymbionts of certain species of Acanthamoeba [42]. Consequently, the proliferation of the host population is likely dependent on a network of microbial interactions between the members of the bacterial and the eukaryotic communities. The host community is also affected by the physicochemical parameters of the environment, such as temperature and chlorine concentrations [30]. For instance, higher concentrations of chlorine are negatively correlated with the presence of protozoa in cooling towers and a temperature higher than $50{ }^{\circ} \mathrm{C}$ is correlated with fewer protozoa counts in water distribution systems [30].

Consequently, the eukaryotic community plays a crucial role in Legionella ecology. This implies that groups of microorganisms affecting the eukaryotic community may have a crucial, but indirect, effect on Legionella ecology in cooling towers or other systems. It also suggests that some specific microbiomes may be more permissive to Legionella survival and growth than others. Indeed, microbiomes with high levels of species interacting positively with Legionella would be more permissive for Legionella growth. Conversely, Legionella would have low survival and growth in a system which microbiome contains mostly species interacting negatively with $\mathrm{Le}$ gionella. So far, the interplay between these communities in the context of Legionella colonization, survival and proliferation in cooling towers is still not well understood.
Furthermore, little research has been done on the entirety of the eukaryotic community in cooling towers. A recent study by Tsao et al. examined the relationship between the protist and bacterial community of three cooling towers [31]. However, this study focused on the protozoan hosts, and did not examine the entirety of the eukaryotic community present in the towers. Previously, we characterized the bacterial communities of 18 cooling towers. Several potential interactions between bacteria and Legionella spp. and L. pneumophila were identified. The presence of Legionella was associated with the presence of several bacterial taxa, such as Brevundimonas, Porphyrobacter and Xanthobacteraceae, but negatively correlated with Pseudomonas [2]. Consequently, we hypothesize that the presence of specific microbial groups could increase permissiveness of the microbiome of cooling towers to L. pneumophila colonization, survival and proliferation through their interactions with $L$. pneumophila's host species. Our objective was to profile the eukaryotic communities of the same 18 cooling towers using an $18 S$ rRNA amplicon sequencing approach. The relationship between the eukaryotic community and the bacterial community was analysed in the context of Legionella ecology, along with the associated physicochemical characteristics of the cooling towers. A network analysis, based on co-occurrence, was performed between the eukaryotic community and the bacterial community to uncover potential interactions between members of the microbial community. The results from the network analysis lead us to investigate the interaction between a bacterial isolate of Brevundimonas sp., L. pneumophila and ciliates hosts species. Furthermore, whole genome sequencing revealed potential mechanisms by which the Brevundimonas isolate favours the growth of L. pneumophila.

\section{Results \\ Sequencing results}

A total of 4,280,578 paired reads were generated from the MiSeq run. The Mothur MiSeq SOP was followed for processing the sequencing data [43]. Quality filtering, denoising and chimera removal of the raw sequences removed a total of 350,988 low-quality sequences, keeping $3,939,401$ sequences. The resulting sequences were then classified using the Bayesian classifier implemented in Mothur and the Silva ribosomal RNA reference database [43-46]. From the classification, 1,891,109 sequences were identified as bacterial sequences, 1029 sequences as Archean and 260,506 sequences as "unknown". Most bacterial sequences were identified as Proteobacteria, mainly Gamma- and Alpha-proteobacteria. The first three most abundant bacterial sequences were identified as an unclassified Gammaproteobacteria sequence, Porphyrobacter and an unclassified Beijerinckiaceae 
sequence. These three most abundant sequences constituted around $60 \%$ of the total bacterial sequences. The bacterial, archaeal and unknown sequences were removed from the data, leaving 1,786,757 eukaryotic sequences (Supplementary Table S1).

The eukaryotic sequences were then clustered into a total 44,183 different OTUs. The counts for each replicate, before and after using the Mothur pipeline, can be viewed in the Supplementary Table S1. For statistical analysis, the OTU counts of each cooling tower sample was created by averaging the OTU read counts of the three replicates. Microbiome analyst was then used for rarefying the data [47]. Before rarefaction, the averaged OTU counts ranged from 3861 counts to 105,815 counts for the different cooling tower samples (Supplementary Table S2). Good's coverage estimator was used to evaluate if the sequencing depth was adequate for diversity analysis. The estimator averaged $97.01 \%$, ranging from a minimum of $90.36 \%$ to a maximum of $99.76 \%$ depending on the sample. The data were filtered, rarefied and normalized to create an OTU table for analysis (see the "Materials and methods" section). This OTU table had 3484 read counts per sample (Supplementary Table S2). The Good's coverage for this rarefied dataset averaged $97.65 \%$ ranging from 96.21 to $99.54 \%$ depending on the sample (Supplementary Table S2).
Furthermore, a blank sample was sequenced in order to determine the presence of any contaminating sequences coming from any steps of the sequencing library preparation used. This blank consisted in running a sterile filter, from the same lot used for the cooling tower samples, through the same DNA extraction protocol and $18 S$ rRNA DNA library protocol as mentioned in the "Materials and methods" section. After sequencing and processing the raw sequence data, the blank contained only 45 sequences, which could be clustered into a total of 15 OTUs. These OTUs were classified as "Unclassified Embryophyta" (plants), when using the Silva ribosomal reference database. This indicates that the cooling tower data were not contaminated by sequencing reads coming from elsewhere.

\section{Eukaryotic profile of cooling towers}

The 18S rRNA gene targeted amplicon sequencing revealed a diverse community of eukaryotes inhabiting the cooling tower environment (Fig. 1a). The characteristics of each tower can be seen in Supplementary Table S3. Overall, the community could be divided into 20 different phyla and classes. Fungal groups were the most abundant and prevalent taxa in the cooling tower samples, with the Basidiomycota and Ascomycota classes being the most dominant (Fig. 1a). For instance, the

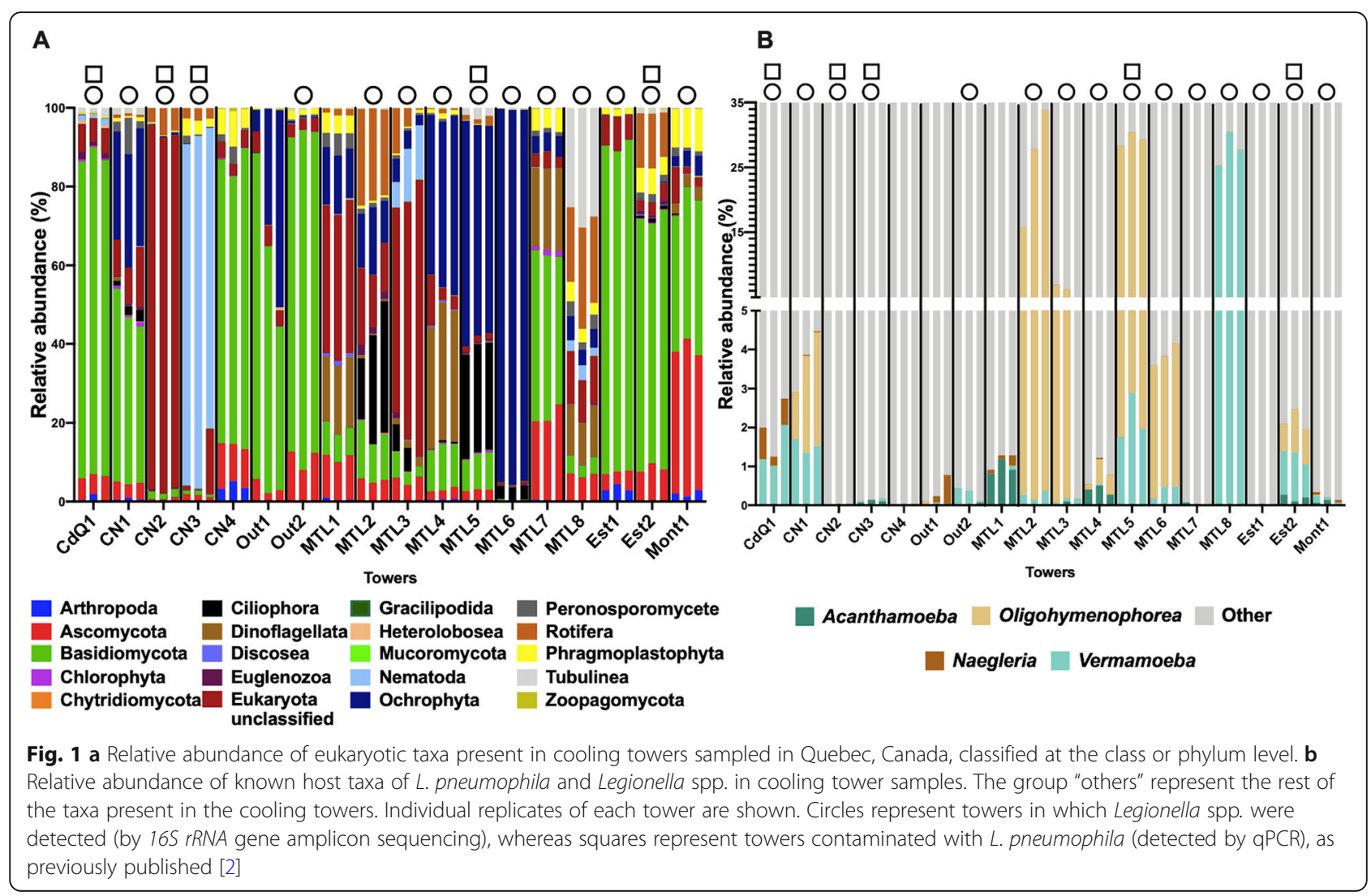


Basidiomycota class dominated (more than $50 \%$ of the community) the eukaryotic community in eight out of the 18 towers (Fig. 1a). Several other fungal groups, such as Zoopagomycota (Zygomycota), Chytridiomycota and Mucoromycota, were detected but at abundances of less than $1 \%$ across all towers sampled. Several taxa comprising known photosynthetic organisms were also detected, such as the Chlorophyta (Microalgae), Dinoflagellates and Ochrophyta. Towers MTL4, MTL5 and MTL6 contained notably high numbers of Ochrophyta. Additionally, micro-animals belonging to the Nematoda and Rotifera taxa were identified in many samples, such as in tower CN3 where nematodes constituted over $80 \%$ of the eukaryotic population. Free-living nematodes are important but underappreciated players in freshwater sediments ecology [48] and could potentially also play an important role in cooling towers. Nevertheless, this high abundance is likely due to their multicellular nature. Macro-eukaryotes were also identified with towers containing sequences related to insects (Arthropoda) and plants (Phragmoplastophyta). For instance, plant-related sequences reached around $10 \%$ of the community in tower Mont1.

Interestingly, several taxa harbouring known host species of L. pneumophila were also present in the towers, including Ciliophora, Discosea, Heterolobosea, Nematoda and Tubulinea (Fig. 1a) [28]. Out of these taxa, we examined the distribution of four of the most important host taxa: the Acanthamoeba genus (Discosea), the Vermamoeba genus (Tubulinea), the Naegleria genus (Heterolobosea) and the Oligohymenophorea class (Ciliophorea) (Fig. 1b). These four taxa contain wellestablished host cell species, such as Acanthamoeba castellanii, Vermamoeba vermiformis, Naegleria fowleri and Tetrahymena pyriformis, respectively [28]. The Nematoda class was not included as a potential host taxon, as it is still unclear whether nematodes actually promote growth or simply ensure survival of L. pneumophila [49, 50]. Since we could, at most, only resolve the OTUs to the genus levels, these groups represent potential hosts of Legionella species, since not all species of these groups may be permissive host cells. The relative abundance of host taxa was less than 5\% in most towers (Fig. $1 b)$; however, the host read counts reached a relative abundance of around 30\% for three towers, MTL2, MTL5 and MTL8 (Fig. 1b).

\section{Alpha diversity and beta diversity are affected by dissolved organic carbon}

Alpha diversity of towers was analysed using the Shannon index and the effect of physicochemical parameters was investigated. Statistical analysis was conducted on the averaged results of the three replicates of each cooling tower sample. Overall, dissolved organic carbon levels (DOC) were positively correlated with alpha diversity (Fig. 2a); however, the correlation between DOC and alpha diversity was modest (spearman's $r_{\mathrm{s}}=0.58, P=$ 0.0056), with DOC following a non-linear regression model $\left(R^{2}=0.43\right)$.

Beta diversity was calculated with the Bray-Curtis dissimilarity index and visualized using non-metric multidimensional scaling plot (NMDS). ANOSIM was used to determine statistical significance and dissimilarity between communities. The beta diversity analysis revealed that DOC levels could partially explain the clustering of the cooling tower communities when using NMDS (Fig. 2b). Thus, communities that had high and low levels of DOC formed distinct clusters. Conversely, towers with medium levels of DOC shared similarity with the other two groups. When comparing only the high and low DOC towers, ANOSIM revealed high dissimilarity between these two groups, with an $R$ value of 0.75 (Supplementary Figure $\mathrm{S} 1$ ). However, the $P$ value was around 0.07 indicating that the two groups were not statistically different from one another.

We hypothesized that a substantial amount of DOC in cooling towers comes from biological contaminants in the air, such as spores and pollen. These contaminants are likely captured by the water droplets and spread in the cooling tower environment. To investigate this, we grouped OTUs likely to produce spores, pollen or seeds, as well as OTUs comprising airborne insects, and plotted them as a function of DOC levels for each tower. This group was named "contaminating OTUs", as these OTUs are mostly comprised of organisms not found in water systems and likely originating from other types of ecosystems, such as green spaces. Consequently, "contaminating" OTUs were the sum of OTUs assigned to the taxa Basidiomycota, Ascomycota, Arthropoda and Phragmoplastophyta, after rarefying the dataset. Interestingly, a modest positive correlation (Spearman $r_{\mathrm{s}}=0.62$, $P=0.003)$ was observed between DOC levels and relative abundance of contaminating OTUs (Fig. 3). Using non-linear regression, the data followed a semi logarithmic curve $\left(R^{2}=0.38\right.$; Fig. 3$)$. Thus, contaminating OTUs seem to contribute to DOC.

\section{Network analysis}

Next, putative ecological relationships between the different taxa of the microbial community of the cooling towers were identified by constructing a microbial ecological network based on co-occurrence (Pearson's correlation) using the MENA pipeline and visualized using Cytoscape 3.7.1 (Fig. 4) [51, 52]. The network was constructed from our previously published bacterial profiling dataset (see [2]) and the eukaryotic community profiles. However, to reduce the number of ecologically non-relevant interactions, the contaminating OTUs were 
A

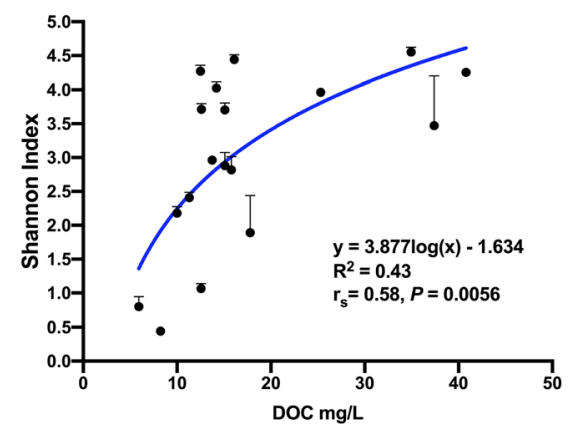

B

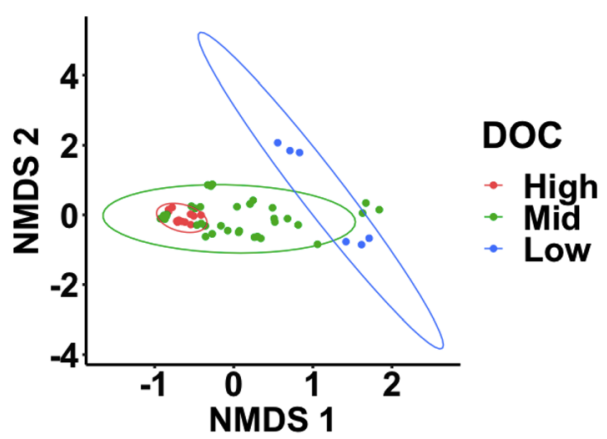

Fig. 2 a Alpha diversity of cooling towers plotted against DOC levels of each tower. A semi-logarithmic curve fit the data best, using non-linear regression. The average and standard deviation of each tower is shown. $\mathbf{b}$ Non-metric multidimensional scaling plot of cooling towers eukaryotic communities categorized by DOC levels and using ANOSIM to evaluate statistical significance of dissimilarity between communities $(R=0.21, P=$ 0.118 , stress $=0.102$ ). The categories are as followed: $<10 \mathrm{mg} / \mathrm{L}$ of DOC were grouped as low; 10 to $20 \mathrm{mg} / \mathrm{L}$ were categorized as mid; 20 to 40 $\mathrm{mg} / \mathrm{L}$ were categorized in the high group. The plot graphs the Bray-Curtis dissimilarity index of each replicate of each tower. Towers with high (red) and low levels (blue) of DOC clustered separately $(R=0.75, P=0.0667$; see Supplementary Figure S1)

removed from the dataset prior to rarefaction for this analysis. As a result, some of the towers were left with less than $75 \%$ of the original sequence counts. Those towers were therefore not considered for the network analysis.

Overall, the network was constituted of 58 nodes and 851 edges (Fig. 4). The general properties of the network revealed that the network did not have scale free or small world properties; however, it showed low modularity $(M=0.128)$ with the presence of 3 modules (Fig. 4$)$ [51]. The genus Legionella could be found in module 2 along with Brevundimonas and Acanthamoeba. Oligohymenophorea, another host taxon of Legionella, was identified in module 3.

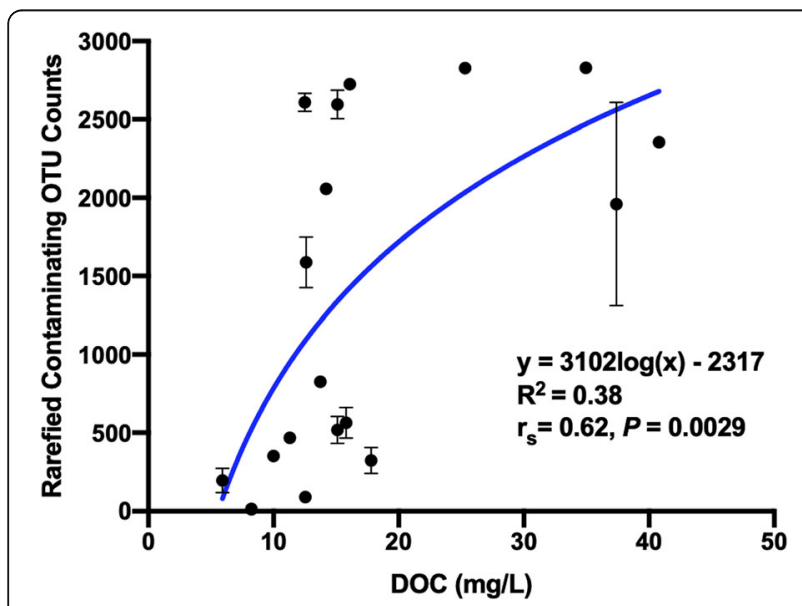

Fig. 3 Rarefied contaminating OTU counts of tower as a function of the respective DOC levels for all sampled cooling towers. The average and standard deviation of each tower is shown. Non-linear regression was used to fit a semi-log curve to the data
To understand the ecological roles of the taxa that constituted each module, the nodes were classified by their within-module $\left(Z_{\mathrm{i}}\right)$ and among-module $\left(P_{i}\right)$ connectivity into peripheral nodes or connector nodes [51, 53]. The data can be visualized in Fig. 4. Peripheral nodes reveal a specialist ecological behaviour, whereas connector nodes indicate a more generalist ecological behaviour [51]. Module 1 consisted of ten connector nodes. These were Rhodobacteraceae, Novosphingobium, Sphingomonadaceae, unclassified Pseudomonadaceae, Methylobacterium, uncultured bacteria groups, Porphyrobacter, Bacillariophytina, Peronosporomycetes and Nevskia. Cytophaga and Acanthamoeba were the only connector nodes of module 2 (containing Legionella). Finally, four connector nodes were found in module 3 . These were Acidovorax, Methylophilaceae, Eustigmatales and Skermanella.

Legionella was classified as a peripheral node within module 2 of the network (Fig. 4). In order to better visualize Legionella's position and role within the network, a sub-network was constructed using the first neighbour nodes of Legionella (Fig. 5a). This subnetwork revealed that Legionella was directly correlated with 26 different taxa. The Legionella neighbours were diverse, including Actinobacteria, Bacteroidetes, Gemmatimonadetes, Nematoda, Protebacteria and algae such as Ochrophyta. Several of these bacterial neighbours were previously identified, using LEfSe, as predictors of varying levels of Legionella in the towers [2]. Finally, several neighbours were connectors nodes, such as Bacillariophytina (diatoms), Cytophaga, Eustigmatales, Porphyrobacter and Rhodobacteraceae. Of note, Cytophaga is the node with the most connection in the network with a total of 41 connections. 


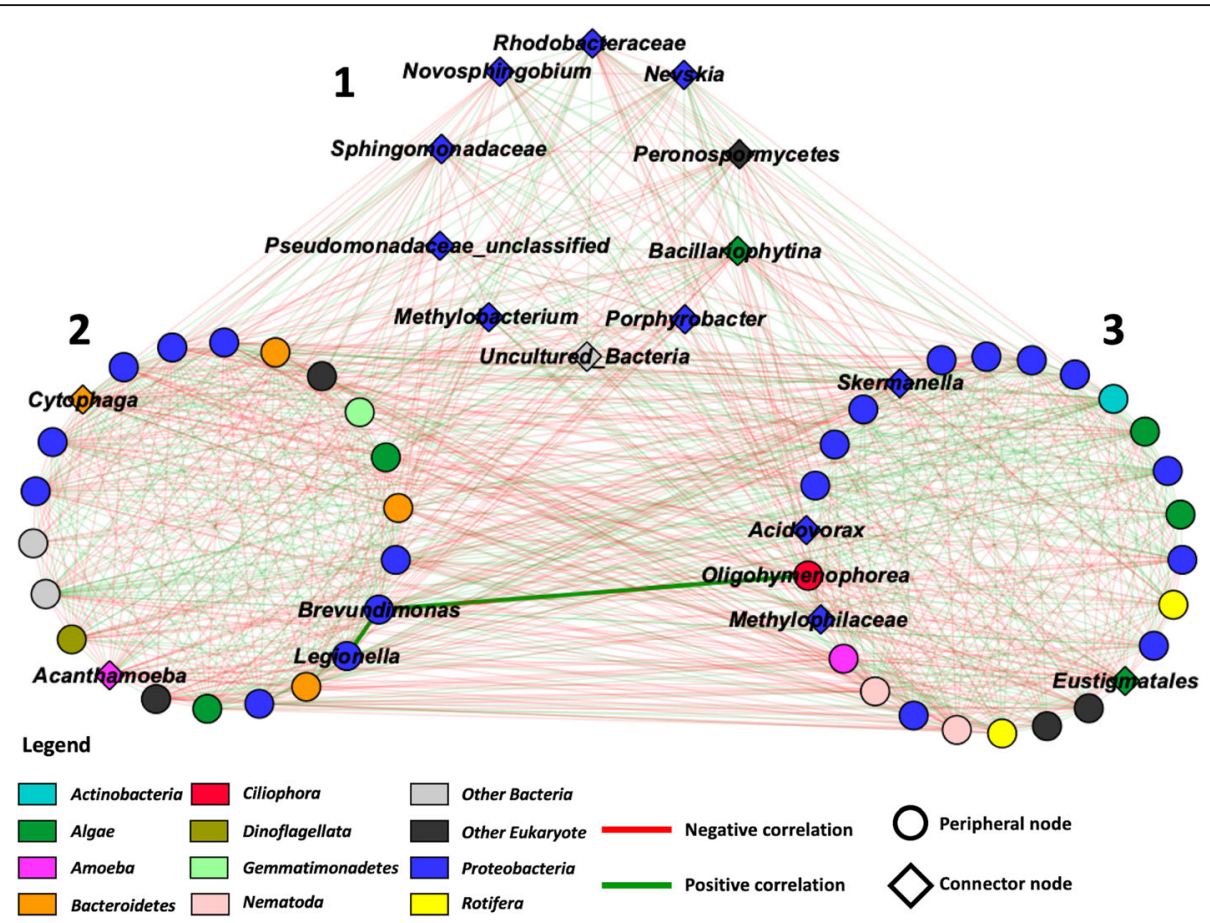

Fig. 4 Microbial ecological network showing correlated taxa (Bacteria and Eukaryotes) in cooling tower samples organised into modules (1 to 3). Green edges represent positive correlations between taxa, and red edges represent negative correlations between taxa. Peripheral and connector nodes are respectively represented by circles and diamonds. A positive correlation can be observed between Brevundimonas, Oligohymenophorea and Legionella, indicated by thicker edges

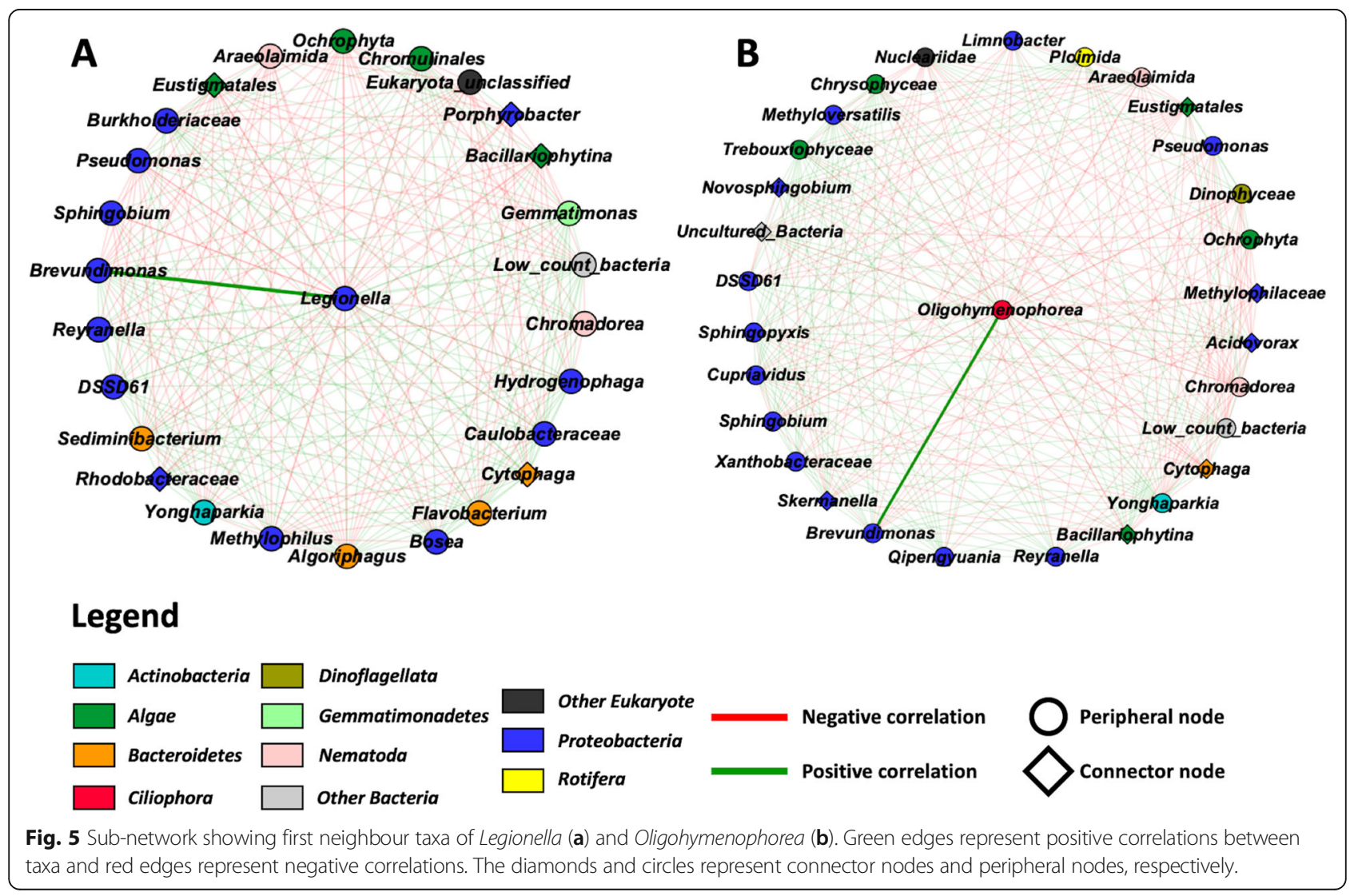


Bacterial predictors of Oligohymenophorea

Eukaryotic profiling revealed that several Legionella host taxa were identified in the tower samples and that some of these taxa were positively correlated with Legionella. Indeed, Oligohymenophorea counts and Vermamoeba counts correlated positively with Legionella counts (Spearman's $r_{\mathrm{s}}=0.72, P=0.0007$ and Spearman's $r_{\mathrm{s}}=$ $0.6, P=0.005$, respectively). Oligohymenophorea is a class of ciliates containing known host species of $L$. pneumophila, such as Tetrahymena pyriformis [21]. Since ciliates are microbial grazers and important in the ecology of Legionella, we sought to identify possible preys of Oligohymenophorea. First, we constructed a sub-network consisting of the first neighbours of Oligohymenophorea (Fig. 5b). Next, we performed a LEfSe analysis on our bacterial dataset to identify bacterial taxa that could predict the presence of Oligohymenophorea in the cooling towers [54]. To this end, we categorized the towers based on the number of rarefied read counts classified as Oligohymenophorea from our eukaryotic dataset. Thus, three groups were created: high $(>100$ counts), low (1 to 100 counts) and absent (0 counts).

Bacterial predictors could be identified for all three Oligohymenophorea level categories (Fig. 6): 19 bacterial taxa were predictive of high levels, four taxa were predictive of low levels, and one genus was predictive of an absence of Oligohymenophorea (Fig. 6). Legionella was the most predictive genus for a high level of the ciliates, whereas Pseudomonas was predictive of an absence of ciliates in the towers. Brevundimonas was also predictive of high levels. Several species were identified by both the network analysis and LEfSe, such as Brevundimonas, Rayranella and Sphingopyxis (Figs. 5b and 6). Moreover, some bacterial predictors of Olygohymenophorea were previously found to be predictors of the presence of $L e$ gionella spp. [2]. For instance, Yonghaparkia, Reyranella, Brevundimonas and Sphingopyxis were predictive of towers containing Legionella [2]. Conversely, Pseudomonas, which correlated negatively with Oligohymenophorea (Fig. 5b), was predictive of towers that did not

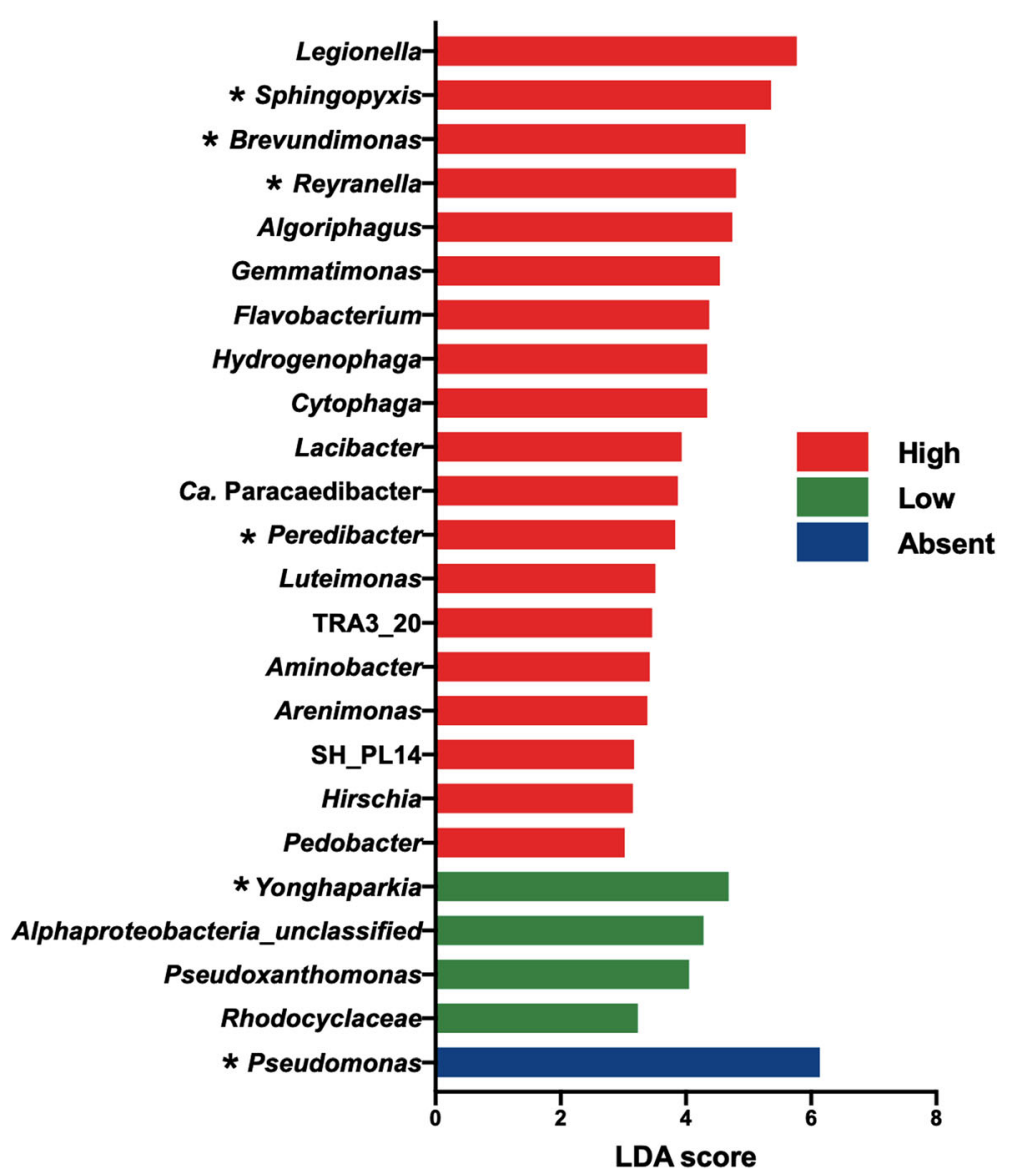

Fig. 6 Bacterial taxa predicting towers containing varying levels of Oligohymenophorea using LEfSe. The towers were classified according to the number of sequences assigned to the Oligohymenophorea class: absent (0 count, blue), low (between 1 and 100, green), high (more than 100, red). Taxa previously identified as predictors of Legionella are indicated with "**" [2] 
have Legionella [2]. Finally, several bacterial predictors were also identified as direct neighbours of Legionella or in the same module as Legionella in the network (Figs. 4 and 5), for instance, Pseudomonas, Brevundimonas, Gemmatimonas, Cytophaga, Flavobacterium and Reyranella.

\section{The bacterial predictor Brevundimonas is a prey for Oligohymenophorea host cells}

The LEfSe analysis revealed that the genus Brevundimonas is a predictor of towers with high levels of Oligohymenophorea. We hypothesized that this correlation between Brevundimonas and Oligohymenophorea, also seen with the network analysis, is probably due to a prey-predator relationship. Brevundimonas SPF441 was isolated from a cooling tower and subjected to whole genome sequencing. The sequencing run generated a total of 500,485 paired reads between 35 and 301 nucleotides in length. After using Trimmomatic (see "Materials and methods" section), a total of 33,119 reads were removed, leaving 467,366 reads. Spades assembled the reads into 66 contigs with a total sequence length of 3 , 201,388 bp. The N50 was 98,818 bp, with the shortest contig at $238 \mathrm{bp}$ and the longest at 344,975 bp. The median depth was calculated at 7.55X. Prokka identified 3161 coding sequences (CDS), 3 rRNA elements and 51 tRNA elements. A short description of the metabolic genes can be viewed in the Supplementary Document DS1. Analysis of the genome with MiGA revealed that our isolate is closely related to Brevundimonas vesicularis with a $95.5 \%$ average nucleotide identity [55]. The $16 S$ RDP classifier implemented in MiGA also showed that the isolate was classified within the Brevundimonas genus. These results indicate that the isolate is most likely a species within the Brevundimonas genus; however, identifying the species would require additional tests.

Given that the network analysis revealed that the genus Brevundimonas was positively correlated with the genus Legionella and the class Olygohymenophorea, coculture experiments were undertaken between the isolated Brevundimonas SPF441 and the ciliates T. pyriformis and $T$. thermophila. The two species of Tetrahymena tested are known host species for L. pneumophila and belong to the Oligohymenophorea class [21, 56]. The Brevundimonas SPF441 counts decreased by 5 logs after $12 \mathrm{~h}$ of co-culture with both Tetrahymena species. In contrast, no decrease in CFU numbers was seen when Brevundimonas SPF441 was incubated alone in the media (Fig. 7a, control). These drastic decreases suggest that Brevundimonas SPF441 is being consumed by Tetrahymena. However, certain Tetrahymena species are known to reject certain species of bacteria they consume by pelletizing them in packages and excreting them from their cells [57]. To test whether or not the Brevundimonas SPF441 cells were being consumed for nutrition, cocultures where performed in Tris buffer, in which Tetrahymena is unable to grow. When fed with Brevundimonas SPF441, T. thermophila number increased by 9 -fold and $T$. pyriformis number increased by 150 -fold over 4 days (Fig. 7b, c). Minimal growth was observed for the ciliates in buffer alone. Our results confirm that this bacterium is readily consumed by the ciliates and is sufficient for growth of the ciliate population.

\section{Brevundimonas SPF441 promotes growth of $L$. pneumophila}

In addition, Brevundimonas could also directly promote the growth of Legionella in water systems. To investigate
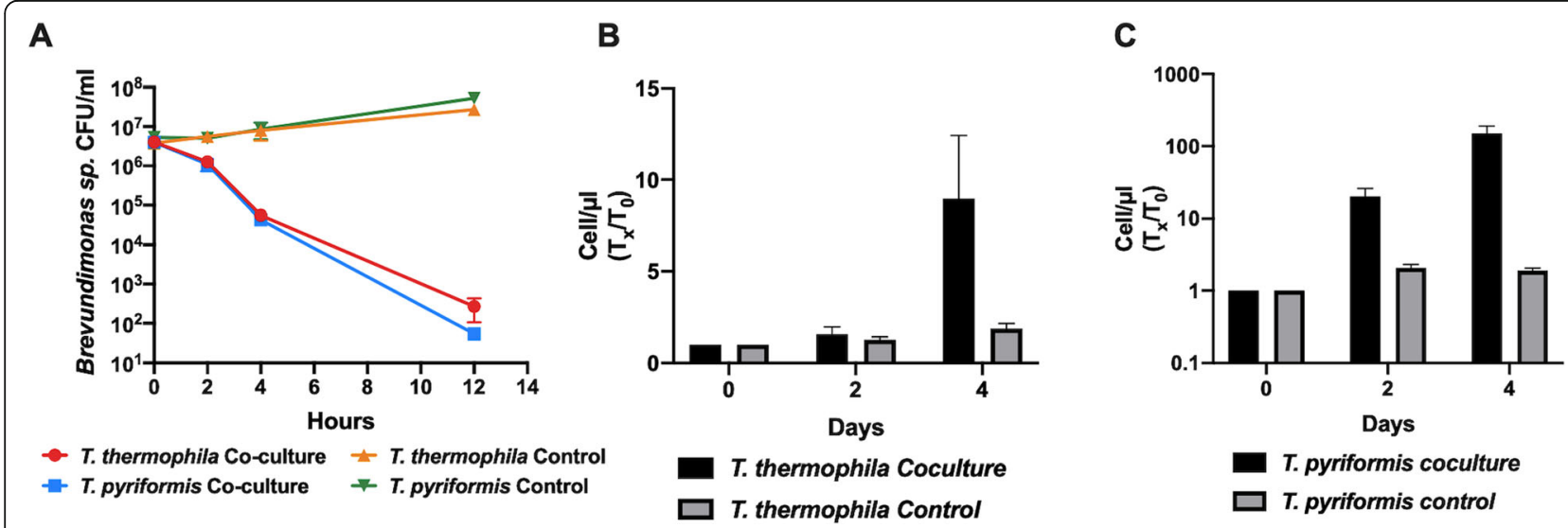

Fig. 7 Brevundimonas SPF441 is a prey for Tetrahymena. Survival of Brevundimonas SPF441 when co-cultured with T. thermophila and T. pyriformis, in plate counting broth (a). Brevundimonas SPF441 suspended alone in plate counting broth was used as the control. Growth $\left(T_{x} / T_{0}\right)$ of $T$. thermophila (b) and T. pyriformis (c) fed with Brevundimonas SPF441, in $10 \mathrm{mM}$ Tris (pH 7.5) and incubated at $30{ }^{\circ} \mathrm{C}$ and $25^{\circ} \mathrm{C}$, respectively. As a control, the two ciliates species were incubated in $10 \mathrm{mM}$ Tris without feeding of Brevundimonas SPF441 at the same temperatures mentioned above 
this possibility, a stimulation assay was performed based on the fact that L. pneumophila requires supplementation of L-cysteine to grow on CYE plates. The assay showed that L. pneumophila grew in a concentric circle around the colony of Brevundimonas SPF441 on plate lacking L-cysteine, which was visualized as a white halo (Fig. 8). This white halo around Brevundimonas SPF441 was not seen on plates not inoculated with L. pneumophila (Fig. 8b). Furthermore, the white halo was confirmed to be L. pneumophila by re-streaking on CYE with L-cysteine (growth) and without L-cysteine (no growth). These results indicate that Brevundimonas SPF441 was able to stimulate the growth of L. pneumophila on CYE plates without L-cysteine. Analysis of the genome of Brevundimonas SPF441 revealed several genes related to cysteine metabolism, such as cystathionine gamma-lyase and cysteine-S-conjugate betalyase (Supplementary Document DS1).

\section{Discussion}

Legionella outbreaks are complex phenomena that are not well understood. The presence of protozoan-host species is crucial for the bacterium's proliferation in the cooling tower environment [19-22]. Consequently, studying the ecology of the host community is key to providing insights into the mechanisms that may lead to high Legionella concentrations in cooling towers. In the present work, we characterized the eukaryotic communities of 18 cooling towers, using an 18S rRNA gene amplicon sequencing approach. The eukaryotic community was analysed in relation to the bacterial and Legionella communities of these same towers, previously identified using a $16 S$ rRNA gene amplicon sequencing approach [2].

\section{Sequencing results}

Although more than 4.2 million reads were sequenced from our library, around $60 \%$ of the data was removed for analysis. The initial denoising steps only removed $8 \%$ of the sequences due to poor quality or presence of chimeras. The amplification and sequencing of bacterial reads caused most of the data loss. Indeed, around 44\% of the reads were classified as bacterial sequences belonging to Proteobacteria, such as Porphyrobacter and unclassified Beijerinckiaceae. After removal of the noneukaryotic sequences, around 1.7 million sequences remained for analysis, constituting about $40 \%$ of the original data. The amplification of a high number of bacterial sequences suggests that the primers used are not specific for eukaryotic organisms. Subsequent to the acquisition of the sequencing data for this project, the Earth Microbiome Project warned that the EukBr reverse primer described in their $18 S$ rRNA protocol can indeed amplify bacterial sequences [58]. Additionally, no bacterial sequences were amplified when we ran a blank through the same pipeline. This indicates that the bacterial sequences were not amplified due to contamination from the kits. Despite the data lost, we estimate that our sequencing depth was adequate to perform subsequent analysis based on Good's coverage (Supplementary Table S2) [31, 59].

\section{Microbial loop in cooling towers}

The complexity and diversity of the ecosystems contained within the towers is evidenced by the presence of multiple trophic levels within the cooling tower environment. Indeed, the presence of primary producers (photoautotrophs, chemoautotrophs), microbial grazers (amoeba, ciliates, nematodes and rotifers) and several

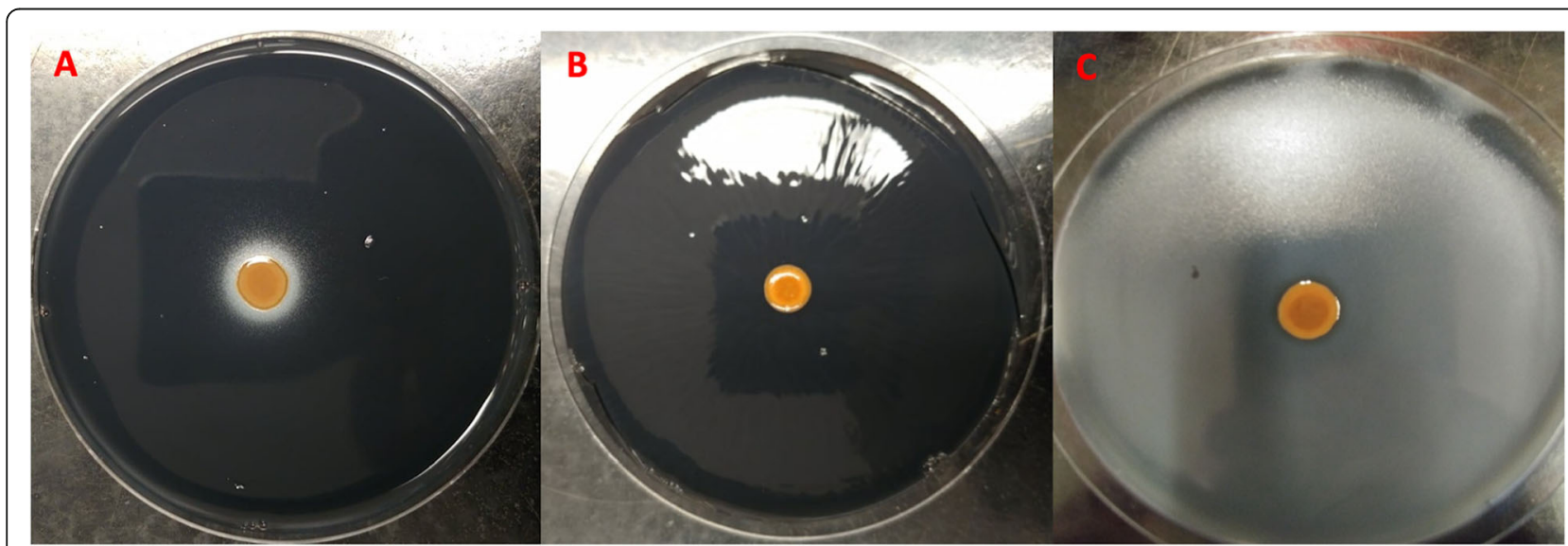

Fig. 8 Bevundimonas SPF441 stimulates growth of L. pneumophila. Stimulation assay was carried on CYE agar without L-cysteine supplementation (a) or with L-cysteine supplementation (c). L. pneumophila was inoculated in soft agar which was poured on the surface of CYE plate (a and $\mathbf{c}$ ). Brevundimonas SPF441 was spotted on each of those plates, and alone as control (b) 
different functional bacterial groups (heterotrophic decomposers, perchlorate reducers, nitrogen fixers, chemolithotrophs) suggests the existence of a local microbial loop within these niches [60]. In this scenario, primary producers, such as the algae Tribouxiophyceae, Ochrophyta, Eustigmatales, Chrysophyceae, Chromulinales in module 2 and 3 or bacterial chemoautotrophs, release dissolved organic carbon (DOC) through waste products and dead cells. Primary production through photosynthesis may be possible as cooling towers are not closed systems, and most have openings that allow light to reach the basin or fill. The DOC is then consumed by the heterotrophic bacterial and fungal populations. Subsequently, the carbon travels up the trophic levels through different groups of microbial grazers (unicellular, such as amoeba and ciliates, then multicellular, such as nematodes and rotifers). The microbial grazers reintroduce the carbon into the cycle in the form of dead cells and waste products. This dynamic indicates that cooling towers not only allow survival of microorganisms but also act as sustainable and active breeding grounds for microorganisms, despite the use of disinfection strategies.

This local microbial loop may impact the Legionella community. Indeed, towers with higher levels of DOC, produced by primary production, may be able to support a higher population of chemoheterotrophic microorganisms. This in turn may promote the establishment of a Legionella host population, as more prey species (either primary producers or chemoheterotrophic microorganisms) may allow for more proliferation of host species. As a result, Legionella could grow more numerously in towers with higher levels of primary production. Moreover, previous research has shown that infected amoeba occur more frequently in cooling towers than natural environments, and that a higher DOC level is a major predictor of infected amoeba by amoeba-associated bacteria, such as Legionella species [29, 61]. Thus, factors controlling the release or uptake of DOC in the cooling tower environment may have important influences on the host cell population. In natural ecosystems, DOC uptake into the trophic chains is usually controlled by grazing activity, by the protozoan population and viral lysis $[62,63]$. However, disinfection schedule is likely another important factor in cooling towers. The use of chlorine and other biocides will cause a certain amount of cellular death, and thus, release DOC into the system [64]. Thus, different effects may be observed for continuous versus periodic applications of disinfectant. Presumably, periodic application could release more DOC by generating peaks of cellular death, but this would require additional studies.

\section{Effect of DOC on cooling tower eukaryotic communities}

In previous work, we observed that higher levels of DOC correlated with lower levels of bacterial alpha diversity, and that this lowered level of alpha diversity was associated with the dominance of Pseudomonas groups [2]. The presence of Pseudomonas groups has been shown to negatively correlate with the presence of $\mathrm{Le}$ gionella $[1,2,65,66]$. In contrast, our results indicated that eukaryotic diversity increased with higher levels of DOC. In the case of beta diversity, high and low DOC samples clustered distinctly, indicating distinct communities. A probable cause for this observation may be the introduction of "contaminating" organisms. By this, we mean the presence of eukaryotic organisms not naturally growing in natural or engineered water systems. Cooling towers intake great volumes of air due to their function and design [67]. This can lead to the presence of airborne fungal spores, fungal tissues, insects, plant tissues and seeds in cooling tower water. Therefore, we defined contaminating OTUs as OTUs belonging to the fungal groups Ascomycota and Basidiomycota, as well as, insects (Arthropoda) and plants (Phragmoplastophyta). Although yeast and moulds were identified, the majority of the fungal groups were associated with macroscopic fungi (mushrooms), usually found in forests. Furthermore, most of the Phragmoplastophyta OTUs were associated with land plants belonging to taxa comprising of grasses and trees. The Arthropods detected were mainly flying insects associated with the Diptera order (flies and mosquitos), but beetles (Coleoptera) were also identified. It is noteworthy that some of the species included in these groups have aquatic larval stages and might therefore be resident of the cooling towers. When we grouped the contaminating OTUs together and plotted the counts as a function of DOC, a modest positive correlation was observed between these two factors. It is noteworthy to mention that this correlation was mainly driven by the Basidiomycota group. Consequently, the results suggest that contaminating OTUs may have some effect on the concentration of DOC within the cooling tower environment, depending on the location of the cooling tower. In this perspective, cooling towers in rural areas or close to green space may receive much more contaminating OTUs than cooling towers in dense urban areas. Other factors surely contribute to DOC concentrations, such as disinfection strategies (as discussed above), source of makeup water, cooling tower design, as well as factors associated with wind, which would affect the presence of contaminating OTUs [67]. Our study suggests that the location and surroundings of a cooling tower may be important to consider when developing a management strategy.

\section{Network analysis and cooling tower ecology}

Network analysis revealed co-occurrence patterns between different taxa in the cooling tower environment (Fig. 4). In our case, the connections between taxa are based on Pearson's correlation. These correlations could 
be due to ecological interactions, such as competition or mutualism, or because they occupy the same niche. One of the main findings of the network analysis was that the cooling tower ecosystem was modular with the identification of three modules within the network. The property of modularity indicates that the network contains a specific number of modules, where a module is a group of taxa that interact mostly with the members of its own module and less with other taxa from other modules in the network [51]. One potential interpretation of the presence of these modules in the network is that they represent distinct sub-niches in the cooling tower ecosystems. However, modularity may also be due to the presence of several phylogenetically related nodes sharing the same optimal niches.

The identification of several connector nodes within the modules may indicate that several taxa may be able to inhabit various niches or that certain niches may overlap. Connector nodes are believed to represent generalist ecological behaviour [51, 53]. For instance, Cytophaga was a connector node identified in module 2. These bacterial species are known to be important consumers of various large organic compounds, such as cellulose and chitin, either through direct consumption of dissolved organic matter or by lysing species containing these compounds, such as cyanobacteria [68]. Correspondingly, we observed that this taxon was connected to several algal nodes from modules 2 and 3. Thus, Cytophaga may be able to inhabit various cooling tower niches containing algal organisms through direct nutritional interactions with these groups. On a side note, the capacity to degrade heavy weight organic compounds has made Cytophaga an important actor of the microbial loop in marine systems [68]. This may indicate that connector nodes are important keystone species, as they could potentially affect the members of several sub-niches.

\section{Microbiome of cooling tower and Legionella ecology}

Our analysis revealed specific trophic interactions that might be important for Legionella ecology. This was clearly recognizable with the interaction between Oligohymenophorea and Brevundimonas identified by the network and LEfSe analyses. Our results showed that bacterial species belonging to the Brevundimonas genus could be used as predictors for identifying towers with high levels of Oligohymenophorea and Legionella [2]. The predator-prey interaction between Tetrahymena and Brevundimonas was confirmed in vitro (Fig. 6). In this scenario, Brevundimonas would be used as a food source for the growth of the ciliate community, which would grow in numbers. This would then allow Legionella species to grow in the cooling tower environment, as they would use these ciliates as host cells. In addition, Oligohymenophorea likely prey on other microorganisms identified with the network analysis and/or LEfSe, such as the bacteria Sphingopyxis, Reyranella, Qipengyuania or Skermanella, and the algae Trebouxiophyceae and Chrysiophyceae, identified with the network analysis. These taxa were previously found to be predictors of the presence of Legionella in cooling towers [2].

Additionally, our results demonstrated that Brevundimonas SPF441 could stimulate the growth of L. pneumophila on CYE agar without L-cysteine supplementation (Fig. 8). This stimulation may be due to the production of essential amino acids, and more specifically production of cysteine, by the Brevundimonas isolate. Indeed, whole genome sequencing identified several genes involved in the synthesis of cysteine or cysteine derivatives in Brevundimonas SPF441. Cystathionine gamma-lyase and cysteine-S-conjugate beta-lyase are both enzymes involved in production of thiocysteine and L-cysteine from L-cystine [69]. Cysteine from the yeast extract in CYE agar is quickly oxidized to L-cystine and cannot be used by $L$. pneumophila, hence the necessity to supplement the CYE medium with L-cysteine [70]. Consequently, Brevundimonas SPF441 may supplement L. pneumophila with an exogenous source of L-cysteine by converting the L-cystine to L-cysteine and thiocysteine. In addition, L. pneumophila is auxotrophic for several amino acids, such as arginine, cysteine, isoleucine, leucine, methionine, threonine and valine [71]. Brevundimonas SPF441 possesses all the genes necessary for the production of these amino acids. Thus, our results suggest that Brevundimonas species may be important for Legionella ecology, through direct and indirect interactions resulting in the promotion of Legionella survival and growth in water systems.

Overall, these results have several implications for Legionella ecology. First, the direct and indirect stimulation of Legionella by Brevundimonas seems to suggest that these two species participate in mutualistic interactions. In the context of a water system, an interesting possibility is that Brevundimonas stimulates the growth of Legionella in order to protect itself from predation by ciliates. Promoting the nearby survival or growth of Legionella may reduce the chance of Brevundimonas from being ingested by predators by reducing the number of predators, as Legionella will kill them. On the other hand, Legionella receives the benefit of a higher chance of survival and proliferation through nutritional supplementation (both through cysteine and host cells). Secondly, the Brevundimonas-Legionella interaction supports the idea that specific groups of organisms, other than host species, are crucial for Legionella ecology. Therefore, some specific microbiomes would be more permissive for colonization, survival and proliferation of Legionella than others. In this perspective, towers with more negatively interacting species would be less permissive (even refractory), whereas 
microbiomes with higher levels of positively interacting species would be more permissive towards Legionella. Nevertheless, understanding which microbiomes would be permissive or not would require additional studies. A potential starting point would be to examine the other positively interacting species from our dataset.

Taken together, our results suggest that ciliates species may be more important than previously thought for Legionella ecology, as most research mainly focuses on the free-living amoeba population [61, 72-74]. The importance of the ciliate community was recently suggested [31]. It is tempting to speculate that the ciliate population may increase the virulence of $L$. pneumophila similarly to what was previously demonstrated in the amoeba A. castellanii [75].

Finally, Pseudomonas is negatively associated with Legionella due to direct or indirect interactions [2]. Our results support the latter possibility, since Pseudomonas correlates negatively with Oligohymenophorea (Fig. 5b) and is a predictor of cooling towers free of Oligohymenophorea (Fig. 6). This could be due to direct killing, since Pseudomonas aeruginosa is able to kill amoeba using its type three secretion system [76]. This may also be true for some species of ciliates, but several Pseudomonas species have also been identified as prey for ciliates, such as Tetrahymena [77, 78]. Thus, more research should go into identifying the mechanisms that promote the presence of Pseudomonas in towers without ciliates.

\section{Conclusion}

In conclusion, our research indicates that the host community is not the single most important factor for Legionella outbreaks, but that instead, Legionella ecology is dependent on various groups of microorganisms. As a result, this may indicate that the microbiomes of cooling towers, which may be more or less permissive for $\mathrm{Le}$ gionella, is a crucial component in Legionella ecology. This permissiveness would be directly related to the species that are present in the microbiomes. As a consequence, our research indicates that Legionella proliferation in cooling tower rely on a network of organisms, many of which have yet to be characterized, representing a "dark web" of interactions. Complex microbial interactions between primary producers, bacterial heterotrophs as well as microbial grazers are seemingly important for Legionella ecology. More specifically, the ciliate community appears to be an important factor to consider for Legionella outbreaks in cooling towers. Furthermore, the role of carbon flux and microbial grazing in the microbial loop may have an important role in $\mathrm{Le}$ gionella ecology. Finally, potential biomarkers for predicting the presence of Legionella and Ciliates were identified using LEfSe. Brevundimonas is an obvious candidate because of its positive relationship with both
Legionella and ciliate hosts. It is warranted to examine the ecological roles of the bacterial biomarkers that were found to predict the presence of Legionella and the host taxa, and how they would come in to play in Legionella ecology. The manipulation of a cooling tower's microbiome to create a non-permissive environment for the colonization by Legionella may be a way to reduce the number of outbreaks of Legionnaires' disease. This could likely be achieved by adjusting operating parameters.

\section{Materials and methods}

\section{Sampling of cooling towers and parameter measurements}

A total of 18 cooling towers were sampled from six different regions in Quebec, Canada, between the 10th and 21st of July 2017. Details were presented in previous work [2]. Briefly, water was sampled in 1-L sterile bottles three times, from the water basin of cooling towers (Supplementary Table S3). The Biomass was collected by filtration $(0.45 \mu \mathrm{m}$ pores $)$ and DNA was extracted using the DNeasy Power water kit from QIAGEN (Cat. No. 14900-100-NF) [2]. The $16 S$ rRNA gene targeted amplicon sequencing using the Illumina MiSeq platform (NCBI Sequence Read Archive accession number PRJNA507738) and the quantification of L. pneumophila using qPCR was reported previously [2].

\section{Eukaryotic community profiling of cooling towers}

18S rRNA amplicon sequencing was performed using the Illumina MiSeq platform (Illumina, Inc.) as described in the Earth Microbiome Project's (EMP) 18S Illumina Amplicon Protocol $[58,79,80]$. This protocol targets the V9 region of the $18 S$ rRNA gene by using primer $1391 \mathrm{~F}$ (5'-GTA CAC ACC GCC CGT C-3') and EukBR (5'TGA TCC TTC TGC AGG TTC ACC TAC-3') [79, 80]. The Illumina two-step indexing protocol was used, where the Illumina overhang adapters were added to the primers described above. The V9 hypervariable region of the $18 S$ rRNA gene was amplified by PCR, using the Paq5000 PCR Hotstart master mix (Agilent Technologies, California, USA) with $10 \mu \mathrm{M}$ of each primer and 2 $\mu \mathrm{l}$ of DNA. The cycling program consisted of an initial denaturation step at $94{ }^{\circ} \mathrm{C}$ for $3 \mathrm{~min}$, followed by $30 \mathrm{cy}$ cles of $94{ }^{\circ} \mathrm{C}$ for $45 \mathrm{~s}, 57^{\circ} \mathrm{C}$ for $60 \mathrm{~s}$ and $72{ }^{\circ} \mathrm{C}$ for $90 \mathrm{~s}$, and a final elongation step of $10 \mathrm{~min}$ at $72{ }^{\circ} \mathrm{C}$. The size of the PCR products $(260 \pm 50 \mathrm{bp})$ was confirmed on a $2 \%$ agarose gel. The PCR products were purified using the Ampure XP bead kit (Beckman Coulter, Indianapolis, IN, USA) following the manufacturer's instructions. The purified PCR products were then indexed using the Nextera XT indexing kit, according to the manufacturer's instructions (Illumina, Inc.). The indexed PCR products were then purified using the Ampure XP bead kit and visualized on a $2 \%$ agarose gel. The purified 
DNA was quantified using the Quant-iT PicoGreen dsDNA assay kit (Thermofisher, MA, USA). The DNA samples were normalized to a concentration of $4 \mathrm{nM}$. The samples were pooled, diluted and denatured with $\mathrm{NaOH}$ to a final concentration of $20 \mathrm{pM}$ in $1 \mathrm{mM}$ $\mathrm{NaOH}$ and HT1 buffer (Illumina, Inc.). This solution was further diluted down to $4 \mathrm{pM}$ in pre-chilled HT1 buffer. Following the same dilution protocol, 4 pM PhiX control (Illumina, Inc.) was produced. The solutions were combined in a microcentrifuge tube to produce a 15\% PhiX spike in, with $90 \mu \mathrm{l}$ of the PhiX solution and $510 \mu \mathrm{l}$ DNA library. This solution was heat denatured for two minutes at $96{ }^{\circ} \mathrm{C}$ and then chilled on ice for 5 min. The sample $(600 \mu \mathrm{l})$ was loaded in a Miseq platform using the 600 cycles MiSeq Reagent Kit v3.

Sequencing data was processed using the Mothur pipeline [81]. Briefly, the paired reads were assembled into contigs. Any contig with ambiguous bases or lengths exceeding $310 \mathrm{bp}$ were culled. The sequences were aligned to the eukaryotic Silva Reference Database release 132 . We customized the database so that it contained only the V9 region of $18 S$ rRNA genes. This provides better alignments and ensures that the reads overlap with the appropriate region of the database. Then, the ends and gaps from the sequence alignment were trimmed so that all sequences had the same alignment coordinates. The sequences were further denoised using a pre-cluster algorithm within Mothur. The resulting unique sequences were purged of chimeras using the VSEARCH algorithm implemented by Mothur. Additionally, any remaining undesirable sequences, such as sequences from Bacteria, Archaea, chloroplasts and mitochondria were removed by, first, classifying the sequences with Bayesian classifier algorithm within Mothur, and, then removing the undesirable sequences. Supplementary table S1 provide the number of sequences left after each key steps of the processing of the data. The sequences were then assigned de novo into OTUs using the cluster.split command with a cutoff of 0.03 . The clustering created a total of 44183 OTUs and a taxonomy file for each OTU. Two intermediate datafiles were created for different analyses or visualization purposes. The first one is an OTU table contained the OTU counts for each replicate (hereafter called table R1 for clarity). The second one was created by averaging the counts for the three replicates of each cooling tower sample (herafter called table A1 for clarity). The average OTU counts for table A1 were rounded down to the closest integer.

Table A1 was mainly used for ecological and statistical analysis, whereas table R1 was mainly used for visualization of each replicate (Fig. 1). Both tables were processed using the Microbiome Analyst [47], which performs data filtration and several ecological analyses, such as community profiling, clustering and biomarker analyses. The low count filter was set so that OTUs are retained only if at least $20 \%$ of their values contain at least 2 counts. This removed a total of 16,736 and 4835 low count OTUs from table R1 and table A1, respectively. Additionally, a default low variance filter, which removes OTU with low variance at $10 \%$ using interquantile range, was used to remove any OTUs that were constant throughout the samples. This removed a total of 75 and 80 OTUs from table R1 and A1, respectively. Next, the tables were rarefied to the minimum library size and total sum scaling was used to normalize the data. Table R1 contained a total of 2951 counts per replicate and table A1 contained a total of 3484 counts per sample. Furthermore, the raw reads of the $18 S$ rRNA amplicon sequencing have been uploaded to NCBI's sequence read archive under the accession number PRJNA563440.

Additionally, a negative control was run separately to determine contamination levels. This blank consisted of running an unused sterile filter through the same pipeline as aforementioned. Thus, the clean filter was processed for DNA extraction, $18 S$ rRNA PCR amplification and sequencing on the MiSeq Illumina platform (V3 reagent kit, 600 cycles). The same mothur pipeline was used to process the raw reads.

\section{Ecological analysis}

Next, the MicrobiomeAnalyst was used to create the taxonomic abundance profiles, the coverage analysis, the alpha and beta diversity analysis and the LEfSe analysis [47].

\section{Good's coverage estimator}

Good's coverage estimator was calculated for each sample using table A1. This was calculated on the unrarefied/unfiltered and rarefied/filtered eukaryotic OTU table (Supplemental Table S2).

\section{Taxonomic abundance profiles}

Table R1 was used to create the taxonomic profile of each replicate. MicrobiomeAnalyst was used to group the OTUs at the phylum level. The host taxa profile was created by creating a count table with the OTUs that were assigned to the genus Acanthamoeba, Naegleria, Oligohymenophorea and Vermamoeba for each replicate. The group "other" was created by subtracting the total counts of the host taxa from the total counts for each replicate. GraphPad prism version 8.3.1 for macOS was used to create the bar graphs and visualize the data.

\section{Alpha diversity analysis}

The Shannon index was calculated for each replicate using table R1. This was done with the microbiome analyst. The data was inputted into GraphPad prism version 
8.3.1 for macOS for statistical testing and visualization. Spearman' rank correlation was conducted on the averaged values of the three replicates for each tower. The average Shannon index of each sample was plotted with error bars showing standard deviation. A semi-log model best fitted the data for the regression analysis. We used a $P$ value cut-off of 0.05 to assess statistical significance for both analyses.

\section{Beta diversity analysis}

Beta diversity was performed on both table A1 and R1. Briefly, the Bray-Curtis index was used to create a dissimilarity matrix for both OTU tables. Non-metric multidimensional scaling was used as an ordination method. The ordination results from table R1 were used for creating the NMDS plot. This was done with $\mathrm{R}$ and the Tidyverse package to visualize the dissimilarity between all replicates [82, 83]. ANOSIM statistical analysis was performed on the ordination results of table A1.

\section{LEfSe analysis}

The LEfSe analysis was performed on the bacterial profiles of the same cooling tower samples we had sequenced in a previous experiment [2]. The raw reads for this bacterial data set have been uploaded on NCBI Sequence Read Archive under the accession number PRJNA507738. Briefly, towers were categorized by the relative abundance of Oligohymenophorea (using table A1): absence (less than 0 read counts per sample), low level (between 1 and 100 read counts per sample) and high level (more than 100 read counts per sample). LEfSe analysis was performed on the resulting OTU table previously described (see [2]). This analysis was conducted through the microbiome analyst but GraphPad prism was used to create the bar plots and visualize the data. We used a $P$ value cut-off of 0.05 for the Kruskal-Wallis test and the Wilcoxon test. The LDA score (log scale) cut-off was set at 3 .

\section{Network construction and analysis}

A network, based on co-occurrence, was constructed between the eukaryotic and bacterial taxa of the cooling towers using the Molecular Ecological Network Analysis pipeline (MENAp) [51]. Briefly, eukaryotic OTUs were regrouped into their respective families using OTU table A1. Bacterial OTUs from the previous study were grouped according to their respective genera [2]. OTUs belonging to the contaminating groups (Ascomycota, $\mathrm{Ba}$ sidiomycota, Phragmoplastophyta and Arthropoda) were removed from the eukaryotic dataset. Towers left with less than $75 \%$ of the initial number of sequences were removed. Therefore, only towers CN1, CN2, CN3, MTL1 to MTL6 and MTL8 were kept for this analysis. The eukaryotic data were rarefied to the sample with the least sequence count (8328 counts per sample). Similarly, the bacterial dataset was rarefied to the smallest sample (22,216 counts per sample). OTUs contributing for less than $0.1 \%$ of the total number of counts were merged together into a group called low count OTU. This was done separately for the bacterial dataset (low count_Bacteria) and the eukaryotic dataset (low_count Eukaryota). The two tables were merged and processed with the MENA pipeline via the following website: http://ieg2.ou.edu/MENA [51]. The network was constructed using the default settings of the pipeline with the exception of the following parameters: the "Majority" setting was set to 1 , the "Logarithm" function was not used and Pearson correlation coefficient was selected to calculate correlations between different OTUs. MENA uses random matrix theory to identify a reliable Pearson's correlation coefficient as a cut-off based on the $x^{2}$ test with Poisson distribution [51, 84]. In our case, MENA identified a Pearson coefficient of 0.32 as the cut-off when using the strictest threshold of $\chi^{2}>0.05$. Cytoscape 3.7.1 was used to visualize the network [52].

\section{Isolation of Brevundimonas sp. from cooling tower}

Bacterial colonies were isolated from a cooling tower on R2A agar and re-streaked three times to ensure pure cultures. Glycerol stock (15\% glycerol in R2A medium) cultures were made for each strain for downstream applications. The identities of morphologically different colonies were determined by sequencing the $16 S$ rRNA gene. Briefly, DNA was extracted from pure cultures using the Wizard genomic DNA purification Kit (Promega). The $16 S$ rRNA gene was amplified by PCR, using bacterial primers 27F (5'-AGAGTTTGATCM TGGCTCAG-3') and 1492R (5'-TACGGYTACCTTGT TACGACTT-3'). The PCR product was cloned into the pGEM-T Easy vector system (Promega). Clones were selected by blue white screening. Plasmids containing the 16S rRNA insert were extracted using a Miniprep plasmid extraction kit (QIAGEN). The insert was sequenced by Sanger sequencing at the Plateforme Génomique de l'Université Laval, Canada. The sequence was then analysed using NCBI BLAST. One of the isolates of interest showed $99.51 \%$ identity with Brevundimonas sp. strain HES1 (Accession MN081030.1). We named the strain Brevundimonas SPF441.

\section{Whole genome sequencing of Brevundimonas SPF441 isolate}

Genomic DNA was extracted from the Brevundimonas SPF441 isolate using the Wizard genomic DNA purification kit (Promega). The genomic DNA quality was verified on a $0.8 \%$ agarose gel and the concentration was determined using the Quant-iT PicoGreen dsDNA assay kit (Thermofisher). The DNA library for whole genome 
sequencing was prepared using the Nextera XT DNA library prep kit (Illumina), according to the manufacturer's instructions. The library was analysed on an Agilent Technology 2100 Bioanalyzer (Agilent) to evaluate proper DNA fragment size. The library was normalized to $2 \mathrm{nM}$ and then pooled together. The pooled library was denatured with $0.2 \mathrm{~N} \mathrm{NaOH}$ and diluted to $12 \mathrm{pM}$ loading concentration with HT1 buffer as per the manufacturer's instructions (Illumina). The library was spiked with PhiX control (20 pM) at $1 \%$. The library was then loaded on the MiSeq sequencing platform (Illumina) with the MiSeq Reagent kit V3 (600 cycles).

A total of 500,485 paired reads were generated. The read quality was evaluated using FastQC [85]. The forward and reverse sequences were processed using Trimmomatic (v0.39) with the following commands: LEADING: 10 TRAI LING: 10 SLIDINGWINDOW: 5: 20 MINLEN: 36 [86]. This removed low-quality reads, leaving 467,366 reads $(93.38 \%$ of initial data). The forward and reverse reads were assembled using SPades (v3.13) [87]. The reads were first corrected using the "only-error-correction" option, and then the corrected reads were assembled using the "only-assembler" option. When assembling the reads, the k-mer length was set to 21, 33, 55, 77, 99 and 127. The assembled genome was uploaded to MiGA (Microbial Genome Atlas, v0.3.12) server, and the NCBI Prok module was used to identify the taxonomy and novelty of the isolate [55]. Bandage was used to infer the quality of the assembly [88]. Additionally, the assembled genome was annotated using Prokka (v1.14) [89], and uploaded to the blastKOALA (v2.2) website to infer metabolic pathways present in the isolate, using the KEGG database [90]. The raw reads of this genome were deposited to NCBI SRA under the Bioproject number PRJNA580507. The Whole Genome Shotgun project for Brevundimonas SPF441 has been deposited at DDBJ/ENA/GenBank under the accession number WJWX00000000. The version described in this paper is version WJWX01000000. This deposited genome was annotated using the NCBI Prokaryotic Genome Annotation Pipeline.

\section{Co-culture of Brevundimonas with Tetrahymena: evaluating the fate of Brevundimonas SPF441}

The fate of Brevundimonas SPF441 when incubated in coculture with Tetrahymena thermophila and Tetrahymena pyriformis was determined by CFU counts. Briefly, $T$. thermophila and T. pyriformis were grown in SPP medium (Sugar Proteose Peptone: $8 \mathrm{~g}$ proteose peptone, $0.8 \mathrm{~g}$ dextrose, $0.4 \mathrm{~g}$ yeast extract and $33 \mathrm{nM} \mathrm{FeCL}$, in 400 of distilled water) at $30{ }^{\circ} \mathrm{C}$ and $21^{\circ} \mathrm{C}$, respectively. Cells were passaged when the density reached $5 \times 10^{5}$ cells $/ \mathrm{ml}$. Twenty-five millilitres of the cell cultures were transferred into $50 \mathrm{ml}$ conical tubes and centrifuged at $600 \mathrm{~g}$ for $5 \mathrm{~min}$. The supernatant was quickly removed and $25 \mathrm{ml}$ of plate counting broth (PCB: $5 \mathrm{~g}$ yeast extract, $10 \mathrm{~g}$ tryptone, $2 \mathrm{~g}$ dextrose, $1 \mathrm{~L}$ water) was added to each tube. One-millilitre aliquots of each ciliate solution were transferred to six wells of two 24-well plates. Six wells on each plate were filled with $1 \mathrm{ml}$ aliquots of sterile PCB to be used as controls. Each well was inoculated with $30 \mu \mathrm{l}$ of a $0.4 \mathrm{OD}_{600 \mathrm{~nm}}$ Brevundimonas SPF441 suspension, resulting in a final inoculum of $4 \times 10^{6} \mathrm{CFU} / \mathrm{ml}$. The co-culture with $T$. thermophila was incubated at $30{ }^{\circ} \mathrm{C}$ while the co-culture with $T$. pyriformis was incubated at $25^{\circ} \mathrm{C}$. CFUs were determined at $0,2,4$ and $12 \mathrm{~h}$ of incubation on nutrient agar. The plates were incubated at $30{ }^{\circ} \mathrm{C}$ for 2 days.

\section{Co-culture of Brevundimonas with Tetrahymena: evaluating growth of Tetrahymena using Brevundimonas SPF441 as food source}

The growth of T.pyriformis and T. thermophila was determined when incubated in Tris buffer and periodically fed with the Brevundimonas SPF441 isolate. Briefly, both ciliates were grown in SPP media as described above to a concentration of $1.0 \times 10^{6}$ cells $/ \mathrm{ml}$. The cells were washed twice in $10 \mathrm{mM}$ tris $(\mathrm{pH}$ 7.5). Tetrahymena cells were counted using a Guava easyCyte flow cytometer, using the FSC and SSC parameters. The cells were then diluted down to $1.0 \times 10^{3}$ cells $/ \mathrm{ml}$ for the co-culture and $1.0 \times$ $10^{4}$ cells $/ \mathrm{ml}$ for the control (ciliates alone), in $25 \mathrm{ml}$ of 10 $\mathrm{mM}$ tris (pH 7.5) solution. Ciliate cultures were counted before inoculation on day 0 and incubated at $25^{\circ} \mathrm{C}$ and 30 ${ }^{\circ} \mathrm{C}$, for T. pyriformis and T. thermophila respectively. Every other day, starting on day 1 of incubation, $200 \mu \mathrm{l}$ of 1.000 $\mathrm{OD}_{600} \mathrm{~nm}$ of Brevundimonas SPF441 isolate culture, washed twice in $10 \mathrm{mM}$ tris solution, was inoculated into the ciliate cultures. Ciliates counts were measured on days 0,2 , and 4 using a Guava easyCyte flow Cytometer.

\section{Stimulation of Legionella pneumophila growth on CYE without L-cysteine}

The stimulation assay was based on Wadowsky and Yee (1983) with slight modification [91]. Briefly, $100 \mu \mathrm{l}$ of 0.2 $\mathrm{OD}_{600 \mathrm{~nm}}$ (around a total of $10^{7} \mathrm{CFU}$ ) of L. pneumophila suspension in AYE was inoculated in $5 \mathrm{ml}$ of soft agar (0.5\% agar). The soft agar was overlaid on CYE without L-cysteine supplementation and on CYE with L-cysteine (control). The agar was left to solidify for 15 to $30 \mathrm{~min}$, after which, $10 \mu \mathrm{l}$ of Brevundimonas isolate, at $0.2 \mathrm{OD}$ $600 \mathrm{~nm}$, was spotted in the middle of the plates. The plates were incubated at $30{ }^{\circ} \mathrm{C}$ for 4 days.

\section{Supplementary information}

Supplementary information accompanies this paper at https://doi.org/10. 1186/s40168-020-00926-6.

Additional file 1: Table S1. Read count for each replicate of every cooling tower sample at the different processing steps. 
Additional file 2: Table S2. Good's coverage estimator for unfiltered and filtered eukaryotic OTU table for each cooling tower sample.

Additional file 3: Table S3. Characteristics of Cooling Tower Samples. Additional file 4: Figure S1. Principal Coordinate Analysis ( $P C O A)$ of cooling towers showing clustering of eukaryotic community according to DOC levels using ANOSIM to evaluate dissimilarity between communities $(R=0.817041, P<0.001)$

Additional file 5: Document DS1. Description of metabolic features in Brevundimonas SPF441.

\section{Acknowledgement}

The authors would like to thank Yves Fontaine and Marie-Ève Benoit for their help with collecting the samples, Jacinthe Mailly for the DOC analysis, and the cooling tower managers. We are indebted to SQI for access to cooling towers and help with sampling.

\section{Authors' contributions}

$\mathrm{KP}, \mathrm{EB}, \mathrm{MP}$ and SPF designed the study. KP performed $18 \mathrm{~S}$ rRNA gene amplicon sequencing, whole genome sequencing and the ciliate co-culture experiments. MH isolated Brevundimonas. DS and FCPC performed the Brevundimonas stimulation assay. KP analysed the data and wrote the first draft of the manuscript. KP, SPF, EB and MP edited the manuscript. All authors approved the submission of the manuscript.

\section{Funding}

This project was funded by a FRQNT Team grant (2016-PR-188813) to SPF and MP and NSERC Discovery Grant (RGPIN/04499-2018) to SPF. Mengqi Hu was funded by a MITACS Globalink award. Fiona Chan Pak Choon received a Rudi Dallenbach Undergraduate Research Award.

\section{Availability of data and materials}

The datasets generated and analysed during the current study are available in the NCBI Sequence Read Archive under the accession PRJNA563440 (18S rRNA gene raw reads), and PRJNA507738 (165 rRNA gene raw reads). The Brevundimonas SPF441 genome was deposited at DDB/ENA/GenBank under the accession WJWX00000000. The version described in this paper is WJWX01000000. The raw reads for this genome were deposited in NCBI sequence read archive under the BioProject number PRJNA580507.

\section{Ethics approval and consent to participate}

Not applicable.

\section{Consent for publication}

Not applicable.

\section{Competing interests}

The authors declare no competing interest.

\section{Author details}

${ }^{1}$ Department of Natural Resource Sciences, Faculty of Agricultural and Environmental Sciences, McGill University, Sainte-Anne-de-Bellevue, QC, Canada. ${ }^{2}$ Department of Civil Engineering, Polytechnique Montreal, Montréal, QC, Canada.

Received: 30 June 2020 Accepted: 20 September 2020

Published online: 12 November 2020

\section{References}

1. Llewellyn AC, Lucas CE, Roberts SE, Brown EW, Nayak BS, Raphael BH, et al. Distribution of Legionella and bacterial community composition among regionally diverse US cooling towers. PLoS One. 2017;12(12):e0189937.

2. Paranjape K, Bédard É, Whyte LG, Ronholm J, Prévost M, Faucher SP. Presence of Legionella spp. in cooling towers: the role of microbial diversity, Pseudomonas, and continuous chlorine application. Water Res. 2020;169: 115252.

3. van Heijnsbergen E, Schalk JA, Euser SM, Brandsema PS, den Boer JW, de Roda Husman AM. Confirmed and potential sources of Legionella reviewed. Environ Sci Technol. 2015;49(8):4797-815.
4. Paschke A, Schaible UE, Hein W. Legionella transmission through cooling towers: towards better control and research of a neglected pathogen. Lancet Respir Med. 2019;7(5):378-80.

5. Alexander TY, Kamali A, Vugia DJ. Legionella epidemiologic and environmental risks. Curr Epidemiol Rep. 2019;6(3):310-20.

6. Fitzhenry R, Weiss D, Cimini D, Balter S, Boyd C, Alleyne L, et al. Legionnaires' disease outbreaks and cooling towers, New York City, New York, USA. Emerg Infect Dis. 2017;23(11):1769.

7. Gallagher G. Disneyland cooling towers linked to Legionnaires' disease outbreak. Infect Dis Children. 2017;30(12):16.

8. Bhopal RS, Fallon RJ, Buist EC, Black RJ, Urquhart JD. Proximity of the home to a cooling tower and risk of non-outbreak Legionnaires' disease. BMJ. 1991;302(6773):378-83.

9. Orkis LT, Harrison LH, Mertz KJ, Brooks MM, Bibby KJ, Stout JE. Environmental sources of community-acquired legionnaires' disease: A review. Int J Hyg Environ Health. 2018;221(5):764-74.

10. Nhu Nguyen TM, llef D, Jarraud S, Rouil L, Campese C, Che D, et al. A community-wide outbreak of legionnaires disease linked to industrial cooling towers - how far can contaminated aerosols spread? J Infect Dis. 2006;193(1):102-11.

11. ECDC. Legionnaires' Disease. ECDC Annual epdimilogical report for 2017. 2019.

12. Dooling KL, Toews K-A, Hicks LA, Garrison LE, Bachaus B, Zansky S, et al. Active bacterial core surveillance for legionellosis-United States, 2011 2013. Morb Mortal Wkly Rep. 2015;64(42):1190-3.

13. Control CfD, Prevention. Legionellosis-United States, 2000-2009. MMWR Morbidity and mortality weekly report. 2011;60(32):1083.

14. National Academies of Sciences $E$, Medicine. Management of Legionella in Water Systems. Washington, DC: The National Academies Press; 2019. p. 304.

15. Shah PP, Barskey AE, Binder AM, Edens C, Lee S, Smith JC, et al. Legionnaires' disease surveillance summary report, United States: 2014-1015. 2018.

16. Armstrong T, Haas CN. A quantitative microbial risk assessment model for Legionnaires' disease: Animal model selection and dose-response modeling. Risk Anal. 2007;27(6):1581-96.

17. Armstrong TW, Haas CN. Legionnaires' disease: evaluation of a quantitative microbial risk assessment model. J Water Health. 2008;6(2):149-66.

18. Buse HY, Schoen ME, Ashbolt NJ. Legionellae in engineered systems and use of quantitative microbial risk assessment to predict exposure. Water Res. 2012;46(4):921-33.

19. Lau H, Ashbolt N. The role of biofilms and protozoa in Legionella pathogenesis: implications for drinking water. J Appl Microbiol. 2009;107(2): 368-78.

20. Rowbotham TJ. Preliminary report on the pathogenicity of Legionella pneumophila for freshwater and soil amoebae. J Clin Pathol. 1980;33(12): 1179-83.

21. Fields B, Shotts E, Feeley J, Gorman G, Martin W. Proliferation of Legionella pneumophila as an intracellular parasite of the ciliated protozoan Tetrahymena pyriformis. Appl Environ Microbiol. 1984;47(3):467-71.

22. Murga R, Forster TS, Brown E, Pruckler JM, Fields BS, Donlan RM. Role of biofilms in the survival of Legionella pneumophila in a model potable-water system. Microbiology. 2001;147(11):3121-6.

23. Isberg RR, O'connor TJ, Heidtman M. The Legionella pneumophila replication vacuole: making a cosy niche inside host cells. Nat Rev Microbiol. 2009;7(1):13

24. Gomez-Valero L, Rusniok C, Carson D, Mondino S, Pérez-Cobas AE, Rolando M, et al. More than 18,000 effectors in the Legionella genus genome provide multiple, independent combinations for replication in human cells. Proc Natl Acad Sci. 2019;116(6):2265-73.

25. Fouque $E$, Trouilhé M-C, Thomas $V$, Hartemann P, Rodier M-H, Héchard Y. Cellular, biochemical, and molecular changes during encystment of freeliving amoebae. Eukaryot Cell. 2012;11(4):382-7.

26. Dobrowsky PH, Khan S, Cloete TE, Khan W. Molecular detection of Acanthamoeba spp., Naegleria fowleri and Vermamoeba (Hartmannella) vermiformis as vectors for Legionella spp. in untreated and solar pasteurized harvested rainwater. Parasit Vectors. 2016;9(1):539.

27. Donlan R, Murga R, Carpenter J, Brown E, Besser R, Fields B. Monochloramine disinfection of biofilm-associated Legionella pneumophila in a potable water model system. Legionella: American Society of Microbiology; 2002. p. 406-10. 
28. Boamah DK, Zhou G, Ensminger AW, O'Connor TJ. From many hosts, one accidental pathogen: the diverse protozoan hosts of Legionella. Front Cell Infect Microbiol. 2017;7:477.

29. Berk S, Gunderson J, Newsome A, Farone A, Hayes B, Redding K, et al. Occurrence of infected amoebae in cooling towers compared with natura aquatic environments: implications for emerging pathogens. Environ Sci Technol. 2006;40(23):7440-4

30. Canals O, Serrano-Suárez A, Salvadó H, Méndez J, Cervero-Aragó S, De Porras VR, et al. Effect of chlorine and temperature on free-living protozoa in operational man-made water systems (cooling towers and hot sanitary water systems) in Catalonia. Environ Sci Pollut Res. 2015;22(9):6610-8.

31. Tsao H-F, Scheikl U, Herbold C, Indra A, Walochnik J, Horn M. The cooling tower water microbiota: seasonal dynamics and co-occurrence of bacterial and protist phylotypes. Water Res. 2019;159:464-79.

32. Rodríguez-Zaragoza S. Ecology of free-living amoebae. Crit Rev Microbiol. 1994;20(3):225-41.

33. Samba-Louaka A, Delafont V, Rodier M-H, Cateau E, Héchard Y. Free-living amoebae and squatters in the wild: ecological and molecular features. FEMS Microbiol Rev. 2019;43(4):415-34.

34. Hahn MW, Höfle MG. Grazing of protozoa and its effect on populations of aquatic bacteria. FEMS Microbiol Ecol. 2001:35(2):113-21.

35. Alsam S, Jeong SR, Sissons J, Dudley R, Kim KS, Khan NA. Escherichia coli interactions with Acanthamoeba: a symbiosis with environmental and clinical implications. J Med Microbiol. 2006;55(6):689-94.

36. Shaheen M, Scott C, Ashbolt NJ. Long-term persistence of infectious Legionella with free-living amoebae in drinking water biofilms. Int J Hyg Environ Health. 2019;222(4):678-86

37. Amaro F, Wang W, Gilbert JA, Anderson OR, Shuman HA. Diverse protist grazers select for virulence-related traits in Legionella. ISME J. 2015;9(7):1607.

38. Kusnetsov JM, Martikainen PJ, Jousimies-Somer HR, Väisänen M-L, Tulkki Al, Ahonen HE, et al. Physical, chemical and microbiological water characteristics associated with the occurrence of Legionella in cooling tower systems. Water Res. 1993;27(1):85-90.

39. Rakić A, Štambuk-Giljanović N. Physical and chemical parameter correlations with technical and technological characteristics of heating systems and the presence of Legionella spp. in the hot water supply. Environ Monit Assess. 2016;188(2):73

40. Richardson I. The incidence of Bdellovibrio spp. in man-made water systems: coexistence with legionellas. J Appl Bacteriol. 1990;69(1):134-40.

41. Abd H, Wretlind B, Saeed A, Idsund E, Hultenby K, SANDSTRÖM G. Pseudomonas aeruginosa utilises its type III secretion system to kill the freeliving amoeba Acanthamoeba castellanii. J Eukaryot Microbiol. 2008;55(3): 235-43.

42. Heinz E, Kolarov I, Kästner C, Toenshoff ER, Wagner M, Horn M. An Acanthamoeba sp. containing two phylogenetically different bacterial endosymbionts. Environ Microbiol. 2007;9(6):1604-9.

43. Kozich JJ, Westcott SL, Baxter NT, Highlander SK, Schloss PD. Development of a dual-index sequencing strategy and curation pipeline for analyzing amplicon sequence data on the MiSeq Illumina sequencing platform. Appl Environ Microbiol. 2013;79(17):5112-20.

44. Quast C, Pruesse E, Yilmaz P, Gerken J, Schweer T, Yarza P, et al. The SILVA ribosomal RNA gene database project: improved data processing and webbased tools. Nucleic Acids Res. 2012;41(D1):D590-D6.

45. Yilmaz P, Parfrey LW, Yarza P, Gerken J, Pruesse E, Quast C, et al. The SILVA and "All-species Living Tree Project (LTP)" taxonomic frameworks. Nucleic Acids Res. 2013;42(D1):D643-D8.

46. Glöckner FO, Yilmaz P, Quast C, Gerken J, Beccati A, Ciuprina A, et al. 25 years of serving the community with ribosomal RNA gene reference databases and tools. J Biotechnol. 2017:261:169-76.

47. Dhariwal A, Chong J, Habib S, King IL, Agellon LB, Xia J. MicrobiomeAnalyst: a web-based tool for comprehensive statistical, visual and meta-analysis of microbiome data. Nucleic Acids Res. 2017:45(W1):W180-W8.

48. Majdi N, Traunspurger W. Free-living nematodes in the freshwater food web: a review. J Nematol. 2015:47(1):28.

49. Brassinga AKC, Kinchen JM, Cupp ME, Day SR, Hoffman PS, Sifri CD. Caenorhabditis is a metazoan host for Legionella. Cell Microbiol. 2010;12(3): 343-61

50. Rasch J, Krüger S, Fontvieille D, Ünal CM, Michel R, Labrosse A, et al. Legionella-protozoa-nematode interactions in aquatic biofilms and influence of Mip on Caenorhabditis elegans colonization. Int J Med Microbiol. 2016;306(6):443-51.
51. Deng $Y$, Jiang $Y-H$, Yang $Y$, He Z, Luo F, Zhou J. Molecular ecological network analyses. BMC Bioinformatics. 2012;13(1):113.

52. Shannon P, Markiel A, Ozier O, Baliga NS, Wang JT, Ramage D, et al. Cytoscape: a software environment for integrated models of biomolecular interaction networks. Genome Res. 2003;13(11):2498-504.

53. Olesen JM, Bascompte J, Dupont YL, Jordano P. The smallest of all worlds: pollination networks. J Theor Biol. 2006;240(2):270-6.

54. Segata N, Izard J, Waldron L, Gevers D, Miropolsky L, Garrett WS, et al. Metagenomic biomarker discovery and explanation. Genome Biol. 2011; 12(6):R60.

55. Rodriguez-R LM, Gunturu S, Harvey WT, Rosselló-Mora R, Tiedje JM, Cole JR, et al. The Microbial Genomes Atlas (MiGA) webserver: taxonomic and gene diversity analysis of Archaea and Bacteria at the whole genome level. Nucleic Acids Res. 2018;46(W1):W282-W8.

56. Kikuhara H, Ogawa M, Miyamoto H, Nikaido Y. Yoshida S-i. Intracellular multiplication of Legionella pneumophila in Tetrahymena thermophila. J UOEH. 1994:16(4):263-75.

57. Trigui H, Paquet VE, Charette SJ, Faucher SP. Packaging of Campylobacter jejuni into multilamellar bodies by the ciliate Tetrahymena pyriformis. Appl Environ Microbiol. 2016;82(9):2783-90.

58. Thompson LR, Sanders JG, McDonald D, Amir A, Ladau J, Locey KJ. A communal catalogue reveals Earth's multiscale microbial diversity. Nature. 2017;551(7681):457-63.

59. Godoy-Vitorino F, Ley RE, Gao Z, Pei Z, Ortiz-Zuazaga H, Pericchi LR, et al. Bacterial community in the crop of the hoatzin, a neotropical folivorous flying bird. Appl Environ Microbiol. 2008;74(19):5905-12.

60. Azam F, Fenchel T, Field J, Grey J, Meyer-Reil L, Thingstad F. The ecological role of water-column microbes. Mar Ecol Prog Ser. 1983;10:257-63.

61. Valster RM, Wullings BA, Bakker G, Smidt H, van der Kooij D. Free-living protozoa in two unchlorinated drinking water supplies, identified by phylogenic analysis of $18 \mathrm{~S}$ rRNA gene sequences. Appl Environ Microbiol. 2009:75(14):4736-46.

62. Fenchel T. The microbial loop-25 years later. J Exp Mar Biol Ecol. 2008; 366(1-2):99-103.

63. Suttle CA. The significance of viruses to mortality in aquatic microbial communities. Microb Ecol. 1994;28(2):237-43.

64. Helmi K, David F, Di Martino P, Jaffrezic M-P, Ingrand V. Assessment of flow cytometry for microbial water quality monitoring in cooling tower water and oxidizing biocide treatment efficiency. J Microbiol Methods. 2018;152:201-9.

65. Stewart CR, Muthye $V$, Cianciotto NP. Legionella pneumophila persists within biofilms formed by Klebsiella pneumoniae, Flavobacterium sp., and Pseudomonas fluorescens under dynamic flow conditions. PLoS One. 2012; 7(11):e50560.

66. Corre $\mathrm{M}-\mathrm{H}$, Delafont V, Legrand A, Berjeaud J-M, Verdon J. Exploiting the richness of environmental waterborne bacterial species to find natural Legionella pneumophila competitors. Front Microbiol. 2019;9(3360).

67. Milosavljevic N, Heikkilä P. A comprehensive approach to cooling tower design. Appl Therm Eng. 2001;21(9):899-915.

68. Kirchman DL. The ecology of Cytophaga-Flavobacteria in aquatic environments. FEMS Microbiol Ecol. 2002;39(2):91-100.

69. Cooper AJ, Pinto J. Cysteine S-conjugate $\beta$-lyases. Amino Acids. 2006;30(1): $1-15$

70. Ewann F, Hoffman PS. Cysteine metabolism in Legionella pneumophila: characterization of an L-cystine-utilizing mutant. Appl Environ Microbiol. 2006:72(6):3993-4000.

71. Eisenreich W, Heuner K. The life stage-specific pathometabolism of Legionella pneumophila. FEBS Lett. 2016:590(21):3868-86.

72. Kuiper MW, Valster RM, Wullings BA, Boonstra H, Smidt H, Van Der Kooij D. Quantitative detection of the free-living amoeba Hartmannella vermiformis in surface water by using real-time PCR. Appl Environ Microbiol. 2006;72(9): 5750-6.

73. Valster RM, Wullings BA, van den Berg R, van der Kooij D. Relationships between free-living protozoa, cultivable Legionella spp., and water quality characteristics in three drinking water supplies in the Caribbean. Appl Environ Microbiol. 2011;77(20):7321-8.

74. Buse HY, Lu J, Struewing IT, Ashbolt NJ. Eukaryotic diversity in premise drinking water using $18 \mathrm{~S}$ rDNA sequencing: implications for health risks. Environ Sci Pollut Res. 2013;20(9):6351-66.

75. Cirillo JD, Falkow S, Tompkins LS. Growth of Legionella pneumophila in Acanthamoeba castellanii enhances invasion. Infect Immun. 1994;62(8): 3254-61. 
76. Matz C, Moreno AM, Alhede M, Manefield M, Hauser AR, Givskov M, et al. Pseudomonas aeruginosa uses type III secretion system to kill biofilmassociated amoebae. ISME J. 2008;2(8):843.

77. Dopheide A, Lear G, Stott R, Lewis G. Preferential feeding by the ciliates Chilodonella and Tetrahymena spp. and effects of these protozoa on bacterial biofilm structure and composition. Appl Environ Microbiol. 2011; 77(13):4564-72.

78. Friman VP, Jousset A, Buckling A. Rapid prey evolution can alter the structure of predator-prey communities. J Evol Biol. 2014;27(2):374-80

79. Amaral-Zettler LA, McCliment EA, Ducklow HW, Huse SM. A method for studying protistan diversity using massively parallel sequencing of $\mathrm{V} 9$ hypervariable regions of small-subunit ribosomal RNA genes. PLoS One. 2009:4(7):e6372

80. Stoeck T, Bass D, Nebel M, Christen R, Jones MD, BREINER HW, et al. Multiple marker parallel tag environmental DNA sequencing reveals a highly complex eukaryotic community in marine anoxic water. Mol Ecol. 2010;19: $21-31$.

81. Schloss PD, Westcott SL, Ryabin T, Hall JR, Hartmann M, Hollister EB, et al. Introducing mothur: open-source, platform-independent, communitysupported software for describing and comparing microbial communities. Appl Environ Microbiol. 2009;75(23):7537-41.

82. Team RC. A language and environment for statistical computing. Vienna: $\mathrm{B}$ Foundation for Statistical Computing; 2012. URL https://www.R-project.org 2019.

83. Wickham H, Wickham MH. Package tidyverse. Easily Install and Load the 'Tidyverse'; 2017.

84. Weiss S, Van Treuren W, Lozupone C, Faust K, Friedman J, Deng Y, et al. Correlation detection strategies in microbial data sets vary widely in sensitivity and precision. ISME J. 2016;10(7):1669-81.

85. Andrews S. FastQC: a quality control tool for high throughput sequence data. Cambridge, United Kingdom: Babraham Bioinformatics, Babraham Institute; 2010

86. Bolger AM, Lohse M, Usadel B. Trimmomatic: a flexible trimmer for Illumina sequence data. Bioinformatics. 2014;30(15):2114-20.

87. Nurk S, Bankevich A, Antipov D, Gurevich A, Korobeynikov A, Lapidus A et al., editors. Assembling genomes and mini-metagenomes from highly chimeric reads. Annual International Conference on Research in Computational Molecular Biology: Springer; 2013.

88. Wick RR, Schultz MB, Zobel J, Holt KE. Bandage: interactive visualization of de novo genome assemblies. Bioinformatics. 2015;31(20):3350-2.

89. Seemann T. Prokka: rapid prokaryotic genome annotation. Bioinformatics. 2014;30(14):2068-9.

90. Kanehisa M, Sato Y, Morishima K. BlastKOALA and GhostKOALA: KEGG tools for functional characterization of genome and metagenome sequences. J Mol Biol. 2016;428(4):726-31.

91. Wadowsky RM, Yee RB. Satellite growth of Legionella pneumophila with an environmental isolate of Flavobacterium breve. Appl Environ Microbiol. 1983;46(6):1447-9

\section{Publisher's Note}

Springer Nature remains neutral with regard to jurisdictional claims in published maps and institutional affiliations.

Ready to submit your research? Choose BMC and benefit from:
- fast, convenient online submission
- thorough peer review by experienced researchers in your field
- rapid publication on acceptance
- support for research data, including large and complex data types
- gold Open Access which fosters wider collaboration and increased citations
- maximum visibility for your research: over 100M website views per year
At BMC, research is always in progress.
Learn more biomedcentral.com/submissions

